\title{
Constraint qualifications for convex inequality systems with applications in constrained optimization
}

\author{
Chong Li $\stackrel{*}{,}$ K. F. Ng † and T. K. Pong ${ }^{\ddagger}$
}

\begin{abstract}
For an inequality system defined by an infinite family of proper convex functions, we introduce some new notions of constraint qualifications in terms of the epigraphs of the conjugates of these functions and study relationships between these new constraint qualifications and other well known constraint qualifications including the basic constraint qualification studied by Hiriart-Urrutty and Lemarechal, Li, Nahak and Singer. Extensions of known results to more general settings are presented, and applications to particular important problems, such as conic programming and approximation theory, are also studied.
\end{abstract}

Key words: Convex inequality system, basic constraint qualification, strong conical hull intersection property, best constrained approximation, conic programming.

AMS(MOS) Subject Classifications. Primary, 90C34; 90C25 Secondary, 52A07; 41A29; 90C46

\section{Introduction}

Many problems in optimization and approximation theory can be recast into one of the following two types: one is a system of convex inequalities

$$
g_{i}(x) \leq 0 \quad \text { for each } i \in I,
$$

and the other is a minimization problem

$$
\begin{array}{ll}
\text { Minimize } & f(x), \\
\text { s. t. } & x \in C, g_{i}(x) \leq 0, i \in I,
\end{array}
$$

where $C$ is a convex set, not necessarily closed. Many authors have studied these two problems with various degrees of generality imposed on the index set $I$, the family of functions $\left\{g_{i}: i \in I\right\}$ or on the underlying space; see for example $[4-6,13-19,21,23,29-36]$ and references therein.

A special case of (1.1) occurs when each $g_{i}$ is the indicator function of a closed convex set $C_{i}$; that is, one considers a family of closed convex sets $\left\{C_{i}: i \in I\right\}$. In [13], Deustch, Li and Ward introduced the notion of the strong conical hull intersection property (the strong CHIP) for a family of finitely many closed convex sets in a Hilbert space in connection with the reformulation of some best approximation problems. Their work was recently extended in $[32,34]$ to the setting of a normed linear space with $I$ being an infinite set.

For the case when $\left\{g_{i}: i \in I\right\}$ is a finite family of continuous convex functions on a finite dimensional vector space, the notion of basic constraint qualification (BCQ) was introduced by Hiriart-Urrutty and

\footnotetext{
*Department of Mathematics, Zhejiang University, Hangzhou 310027, P. R. China, (cli@zju.edu.cn). This author was supported in part by the National Natural Science Foundation of China (grant 10671175) and Program for New Century Excellent Talents in University.

${ }^{\dagger}$ Department of Mathematics, Chinese University of Hong Kong, Hong Kong, P. R. China, (kfng@math.cuhk.edu.hk). This author was supported by a direct grant (CUHK) and an Earmarked Grant from the Research Grant Council of Hong Kong.

${ }^{\ddagger}$ Department of Mathematics, Chinese University of Hong Kong, Hong Kong, P. R. China, (tkpong@gmail.com).
} 
Lemarechal (see [19]). The notion was extended to cover the case of infinite family of continuous convex functions with a continuous sup-function $\sup _{i \in I} g_{i}$ by Li, Nahak and Singer (see [36]), who also studied many aspects of $\mathrm{BCQ}$ in relation to other constraint qualifications. Recall from $[32,33,36]$ that the inequality system (1.1) is said to satisfy the BCQ at $x \in S:=\left\{x \in X: g_{i}(x) \leq 0, i \in I\right\}$ if

$$
N_{S}(x)=\text { cone } \bigcup_{i \in I(x)} \partial g_{i}(x),
$$

(see the next section for notations and definitions). The concept of BCQ relative to $C$ was introduced in [31-33] in order to take care of the abstract constraint set $C$. In these papers, under some continuity assumption as the one used in [36], the system (1.1) with the family $\left\{\delta_{C} ; g_{i}, i \in I\right\}$ was considered in place of $\left\{g_{i}: i \in I\right\}$.

Constraint qualifications involving epigraphs (first introduced in $[8,9]$ ) have been extensively used by many authors (see for example $[4-10,15,16,24-29,35]$ ). In particular in connection with the study of a conic programming problem (see Example 2.1 below), Jeyakumar et al. [25, 26,29] and Bot et al. [7] studied several new constraint qualifications (such as what they called the condition $\left(\mathbf{C}^{*}\right)$ and the CCCQ, see $[26,29]$ for their definitions). Inspired by these works as well as that of Dinh, Goberna and López in [15] (especially in regard with the new optimality conditions for (1.2)), we define the following concept: the inequality system (1.1) is said to have the conical epigraph hull property (conical EHP) if

$$
\text { epi } \sigma_{S}=\text { cone } \bigcup_{i \in I} \operatorname{epi} g_{i}^{*},
$$

where $S=\left\{x: g_{i}(x) \leq 0, \forall i \in I\right\}$. In particular, (1.3) reduces to the SECQ introduced in [35] if $g_{i}=\delta_{C_{i}}$ for some family of closed convex sets $\left\{C_{i}: i \in I\right\}$. We show that by suitably choosing the family $\left\{g_{i}: i \in I\right\}$, the conical EHP reduces to the CCCQ defined in $[7,26]$. In section 4 , we derive some relationships between the EHP, the BCQ and the Pshenichyni-Levin-Valadier property (PLV property). We also give some applications involving the strong CHIP and the convex Farkas-Minkowski systems (studied by Li, Nahak and Singer in [36]).

In this paper, we consider (1.2) under minimal assumptions: $f$ is a proper convex lower semicontinuous function and that $\left\{g_{i}: i \in I\right\}$ is a family of proper convex functions (not necessarily lower semicontinuous) defined on a locally convex Hausdorff topological vector space $X$ with proper sup-function, where $I$ is an arbitrary index set. The last three sections of this paper are on applications of results obtained in section 4. An optimality condition (of Lagrange type) for (1.2) is established in section 5 and as a consequence we provide an improved version of [16, Theorem 3] on a characterization of minimizers for the problem (1.2); our argument differs from [16] and allows us to treat the case when each $g_{i}$ is not necessarily lower semicontinuous. In particular, our results here cover the interesting conic programming case in which the feasible solution set is not necessarily closed (as the involved functions are not necessarily lower semicontinuous). Several known results in the conic programming problem (see $[24,26,29]$ ) are extended/improved in section 6 . Finally, we study a best approximation problem in section 7 .

\section{Notations and preliminary results}

The notation used in the present paper is standard (cf. $[11,19,40])$. In particular, we assume throughout the whole paper (unless otherwise specified) that $X$ is a real locally convex Hausdorff topological vector space and let $X^{*}$ denote the dual space of $X$, whereas $\left\langle x^{*}, x\right\rangle$ denotes the value of a functional $x^{*}$ in $X^{*}$ at $x \in X$, i.e., $\left\langle x^{*}, x\right\rangle=x^{*}(x)$. Let $A$ be a set in $X$. The interior (resp. closure, convex hull, convex cone hull, linear hull, affine hull, boundary) of $A$ is denoted by int $A(r e s p . \bar{A}, \operatorname{co} A, \operatorname{cone} A, \operatorname{span} A$, aff $A$, bd $A$ ). The positive polar cone $A^{\oplus}$ and the negative polar cone $A^{\ominus}$ are defined respectively by

$$
A^{\oplus}:=\left\{x^{*} \in X^{*}:\left\langle x^{*}, z\right\rangle \geq 0 \text { for all } z \in A\right\}
$$


and

$$
A^{\ominus}:=\left\{x^{*} \in X^{*}:\left\langle x^{*}, z\right\rangle \leq 0 \text { for all } z \in A\right\} .
$$

The normal cone of $A$ at $z_{0} \in A$ is denoted by $N_{A}\left(z_{0}\right)$ and is defined by $N_{A}\left(z_{0}\right)=\left(A-z_{0}\right)^{\ominus}$. The indicator function $\delta_{A}$ and the support function $\sigma_{A}$ of $A$ are respectively defined by

$$
\delta_{A}(x):=\left\{\begin{array}{cc}
0, & x \in A, \\
\infty, & \text { otherwise }
\end{array}\right.
$$

and

$$
\sigma_{A}\left(x^{*}\right):=\sup _{x \in A}\left\langle x^{*}, x\right\rangle \text { for each } x^{*} \in X^{*} .
$$

Let $f$ and $g$ be proper functions respectively defined on $X$ and $X^{*}$. Let $f^{*}, g^{*}$ denote their conjugate functions, that is

$$
\begin{aligned}
f^{*}\left(x^{*}\right) & :=\sup \left\{\left\langle x^{*}, x\right\rangle-f(x): x \in X\right\} \quad \text { for each } x^{*} \in X^{*}, \\
g^{*}(x) & :=\sup \left\{\left\langle x^{*}, x\right\rangle-g\left(x^{*}\right): x^{*} \in X^{*}\right\} \quad \text { for each } x \in X .
\end{aligned}
$$

The epigraph of a function $f$ on $X$ is denoted by epi $f$ and defined by

$$
\text { epi } f:=\{(x, r) \in X \times \mathbb{R}: f(x) \leq r\} .
$$

For a proper convex function $f$, the subdifferential of $f$ at $x \in X$, denoted by $\partial f(x)$, is defined by

$$
\partial f(x):=\left\{x^{*} \in X^{*}: \quad f(x)+\left\langle x^{*}, y-x\right\rangle \leq f(y) \quad \text { for each } y \in X\right\} .
$$

Moreover, the Young's equality holds (cf. [40, Theorem 2.4.2 (iii)]):

$$
f(x)+f^{*}\left(x^{*}\right)=\left\langle x^{*}, x\right\rangle \text { if and only if } x^{*} \in \partial f(x) .
$$

In particular,

$$
\left(x^{*},\left\langle x^{*}, x\right\rangle-f(x)\right) \in \operatorname{epi} f^{*} \quad \text { for each } x^{*} \in \partial f(x) .
$$

We also define

$$
\operatorname{im} \partial f:=\left\{y^{*} \in X^{*}: y^{*} \in \partial f(x) \text { for some } x \in X\right\}
$$

and

$$
\operatorname{dom} \partial f:=\{x \in X: \partial f(x) \neq \emptyset\} .
$$

For a convex subset $A$ of $X$, the following statements are standard and easily verified:

$$
\begin{gathered}
\sigma_{A}=\delta_{A}^{*}, \quad N_{A}(x)=\partial \delta_{A}(x) \quad \text { for each } x \in A, \\
\sigma_{A}\left(x^{*}\right)=\left\langle x^{*}, x\right\rangle \Leftrightarrow x^{*} \in N_{A}(x) \Longleftrightarrow\left(x^{*},\left\langle x^{*}, x\right\rangle\right) \in \operatorname{epi} \sigma_{A} \quad \text { for each }\left(x, x^{*}\right) \in A \times X^{*} .
\end{gathered}
$$

Moreover, for each $\left(x^{*}, \alpha\right) \in X^{*} \times \mathbb{R}$,

$$
\left(x^{*}, \alpha\right) \in \operatorname{epi} \sigma_{A} \Longleftrightarrow\left\langle x^{*}, x\right\rangle \leq \alpha \quad \text { for each } x \in A .
$$

Let $\left\{A_{i}: i \in J\right\}$ be a family of subsets of $X$ containing the origin. The set $\sum_{i \in J} A_{i}$ is defined by

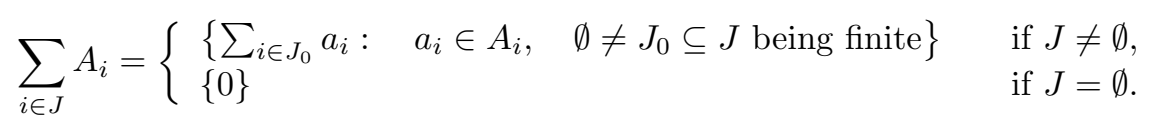

In the remainder of this paper, let $\left\{g_{i}: i \in I\right\}$ denote a family of proper convex functions on $X$, where $I$ is an index set. Let $G$ denote the sup-function of $\left\{g_{i}: i \in I\right\}$, that is,

$$
G(x):=\sup \left\{g_{i}(x): i \in I\right\} \quad \text { for each } x \in X .
$$


We always assume that the sup-function is proper. Let $S$ denote the solution set of the inequality system (1.1) defined by $\left\{g_{i}: i \in I\right\}$, that is,

$$
S:=\left\{x: g_{i}(x) \leq 0, \forall i \in I\right\}=\{x: G(x) \leq 0\} .
$$

For each $x \in X$, we define

$$
I(x)=\left\{i \in I: g_{i}(x)=G(x)=0\right\}
$$

and

$$
\tilde{I}(x):=\left\{i \in I: g_{i}(x)=G(x)\right\} .
$$

The consideration of optimization problem (1.2) abounds in the literature. We end this section with one such example (which will be discussed in detail in section 6). Consider the following conic programming problem that has been studied in [5], and has also been studied in [2,7,24-29] for the special case when $X, Z$ are Banach spaces and $g: X \rightarrow Z$ is $K$-convex continuous.

Example 2.1. Suppose that $X, Z$ are locally convex Hausdorff topological vector spaces, $C \subseteq X$ is a convex set and $K \subseteq Z$ is a closed convex cone. Define an order on $Z$ by saying $y \leq_{K} x$ if $y-x \in-K$. We attach a greatest element $\infty$ with respect to $\leq_{K}$ and denote $Z \bullet:=Z \cup\{+\infty\}$. The following operations are defined on $Z^{\bullet}$ : for any $z \in Z, z+\infty=\infty+z=\infty$ and $t \infty=\infty$ for all $t \geq 0$.

Consider the following conic programming problem:

$$
\begin{array}{ll}
\text { Minimize } & f(x), \\
\text { s. t. } & x \in C, g(x) \in-K,
\end{array}
$$

where $f: X \rightarrow \mathbb{R} \cup\{+\infty\}$ is a proper convex lower semicontinuous function and $g: X \rightarrow Z \bullet$ is $K$-convex in the sense that for every $u, v \in X$ and every $t \in[0,1]$,

$$
g(t u+(1-t) v) \leq_{K} t g(u)+(1-t) g(v),
$$

(see [2, 4, 5, 22, 25]). As in [4], we define for each $\lambda \in K^{\oplus}$,

$$
(\lambda g)(x):= \begin{cases}\langle\lambda, g(x)\rangle & \text { if } x \in \operatorname{dom} g \\ +\infty & \text { otherwise }\end{cases}
$$

where $\operatorname{dom} g:=\{x \in X: g(x) \in Z\}$. It is easy to see that $g$ is $K$-convex if and only if $(\lambda g)(\cdot): X \rightarrow$ $\mathbb{R} \cup\{+\infty\}$ is a convex function for each $\lambda \in K^{\oplus}$. The problem (2.6) can be equivalently stated as

$$
\begin{array}{ll}
\text { Minimize } & f(x), \\
\text { s. } t . & \delta_{C}(x) \leq 0,(\lambda g)(x) \leq 0 \text { for each } \lambda \in K^{\oplus} .
\end{array}
$$

Thus (2.6) can be viewed as an example of (1.2) by letting $I=K^{\oplus} \cup\left\{i_{0}\right\}$ with $i_{0} \notin I$ and

$$
g_{i_{0}}=\delta_{C}, g_{\lambda}=\lambda g \quad \text { for each } \lambda \in K^{\oplus} .
$$

\section{The Basic Constraint Qualification}

We begin with the following definitions adopted from $[32,36]$. In the remainder, we shall adopt the convention that cone $A=\{0\}$ when $A$ is an empty set.

Definition 3.1. Let $C$ be a convex set in $X$. The family $\left\{g_{i}: i \in I\right\}$ is said to satisfy

(i) the Pshenichyni-Levin-Valadier (the PLV) property at $x \in X$ if

$$
\partial G(x)=\operatorname{co} \bigcup_{i \in \tilde{I}(x)} \partial g_{i}(x)
$$


(ii) the basic constraint qualification (the $B C Q$ ) at $x \in S$ if

$$
N_{S}(x)=\text { cone } \bigcup_{i \in I(x)} \partial g_{i}(x)
$$

(ii') the $B C Q$ relative to $C$ at $x \in C \cap S$ if

$$
N_{C \cap S}(x)=N_{C}(x)+\text { cone } \bigcup_{i \in I(x)} \partial g_{i}(x)
$$

(iii) the PLV property (resp. the BCQ, the BCQ relative to $C$ ) if (3.1) (resp. (3.2), (3.3)) holds for each $x \in X$ (resp. $x \in S, x \in C \cap S$ ).

Remark 3.1. In [16, Definition 2] (under the assumption that each $g_{i}$ is lower semicontinuous), the property that the family $\left\{g_{i}: i \in I\right\}$ satisfies the $B C Q$ relative to $C$ was also described as the system $\left\{\delta_{C} ; g_{i}, i \in I\right\}$ is locally Farkas-Minkowski.

A relationship between the notions (ii) and (ii) ${ }^{\prime}$ in Definition 3.1 is shown in the following proposition.

Proposition 3.1. Consider a convex set $C$ and $x \in C \cap S$. Then the family $\left\{g_{i}: i \in I\right\}$ satisfies the $B C Q$ relative to $C$ at $x$ if and only if the family $\left\{\delta_{C} ; g_{i}, i \in I\right\}$ satisfies the $B C Q$ at $x$. Consequently, the family $\left\{g_{i}: i \in I\right\}$ satisfies the $B C Q$ relative to $C$ if and only if the family $\left\{\delta_{C} ; g_{i}, i \in I\right\}$ satisfies the $B C Q$.

Proof. Take $j \notin I$ and set $g_{j}:=\delta_{C}$. Writing $J:=I \cup\{j\}$, the family $\left\{\delta_{C} ; g_{i}, i \in I\right\}$ becomes $\left\{g_{i}: i \in J\right\}$ such that $C \cap S=\left\{y \in X: g_{i}(y) \leq 0, \forall i \in J\right\}$ and $J(x)=\{j\} \cup I(x)$, where for $x \in C \cap S$,

$$
J(x):=\left\{i \in J: g_{i}(x)=\max \left\{\sup _{i \in I} g_{i}(x), \delta_{C}(x)\right\}=0\right\} .
$$

Then by $(2.3)$,

$$
N_{C}(x)+\text { cone } \bigcup_{i \in I(x)} \partial g_{i}(x)=\text { cone } \bigcup_{i \in J(x)} \partial g_{i}(x) .
$$

Thus the first assertion follows. The second follows immediately from the first.

Remark 3.2. (i) We have

$$
\partial G(x) \supseteq \operatorname{co} \bigcup_{i \in \tilde{I}(x)} \partial g_{i}(x) \quad \text { for each } x \in X .
$$

Indeed, let $i \in \tilde{I}(x)$ and $y^{*} \in \partial g_{i}(x)$. Then $g_{i}(x)=G(x)$. Since $y^{*} \in \partial g_{i}(x)$ and $g_{i}$ is proper, $g_{i}(x) \neq+\infty$. Now it follows that

$$
\left\langle y^{*}, y-x\right\rangle \leq g_{i}(y)-g_{i}(x) \leq G(y)-G(x) \quad \text { for each } y \in X .
$$

This shows that $y^{*} \in \partial G(x)$ and so (3.4) is proved. Thus, the family $\left\{g_{i}: i \in I\right\}$ has the PLV property at $x \in X$ if and only if

$$
\partial G(x) \subseteq \operatorname{co} \bigcup_{i \in \tilde{I}(x)} \partial g_{i}(x) .
$$

Hence, the family $\left\{g_{i}: i \in I\right\}$ has the PLV property if and only if (3.6) holds for each $x \in \operatorname{dom} \partial G$.

(ii) If $i \in I(x)$ and $y^{*} \in \partial g(x)$, then $G(x)=0$. It follows from (3.5) that $y^{*} \in N_{S}(x)$. Thus

$$
N_{S}(x) \supseteq \text { cone } \bigcup_{i \in I(x)} \partial g_{i}(x) \quad \text { for each } x \in S .
$$

Therefore, the family $\left\{g_{i}: i \in I\right\}$ satisfies the $B C Q$ at $x \in S$ if and only if

$$
N_{S}(x) \subseteq \text { cone } \bigcup_{i \in I(x)} \partial g_{i}(x)
$$


(iii) Note that if $x \in \operatorname{int} S$, then $N_{S}(x)=\{0\}$. Recalling our convention cone $\emptyset=\{0\}$ (see Definition 3.1 (ii)), it follows from (3.7) that

$$
\{0\}=N_{S}(x)=\text { cone } \bigcup_{i \in I(x)} \partial g_{i}(x) \quad \text { for each } x \in \operatorname{int} S .
$$

Hence, the family $\left\{g_{i}: i \in I\right\}$ satisfies the BCQ if and only if (3.8) holds for each $x \in S \backslash$ int $S$.

(iv) Applying parts (ii) and (iii) to the family of functions $\left\{\delta_{C} ; g_{i}, i \in I\right\}$ in place of $\left\{g_{i}: i \in I\right\}$ and invoking Proposition 3.1, we obtain that the family $\left\{g_{i}: i \in I\right\}$ satisfies the BCQ relative to $C$ at $x$ if and only if we have

$$
N_{C \cap S}(x) \subseteq N_{C}(x)+\text { cone } \bigcup_{i \in I(x)} \partial g_{i}(x),
$$

and that the family $\left\{g_{i}: i \in I\right\}$ satisfies the $B C Q$ relative to $C$ if and only if (3.9) holds for each $x \in(C \cap S) \backslash \operatorname{int}(C \cap S)$.

Recall from $[13,32,34]$ that a family of convex sets $\left\{C_{i}: i \in I\right\}$ is said to have the strong conical hull intersection property (the strong CHIP) at $x \in \bigcap_{i \in I} C_{i}$ if

$$
N_{\bigcap_{i \in I} C_{i}}(x)=\sum_{i \in I} N_{C_{i}}(x) .
$$

If (3.10) holds for every $x \in \bigcap_{i \in I} C_{i}$, then we say that the family has the strong CHIP.

Proposition 3.2. Let $x \in C \cap S$ and suppose that the family $\left\{g_{i}: i \in I\right\}$ satisfies the $B C Q$ at $x$. Then $\{C, S\}$ has the strong CHIP at $x$ if and only if the family $\left\{g_{i}: i \in I\right\}$ satisfies the BCQ relative to $C$ at $x$.

Proof. By the given assumption, (3.2) holds. Hence we have the following equivalences:

$$
\begin{aligned}
\{C, S\} \text { has the strong CHIP at } x & \Leftrightarrow N_{C \cap S}(x)=N_{C}(x)+N_{S}(x) \\
& \Leftrightarrow N_{C \cap S}(x)=N_{C}(x)+\text { cone } \bigcup_{i \in I(x)} \partial g_{i}(x) \\
& \Leftrightarrow\left\{g_{i}: i \in I\right\} \text { satisfies the BCQ relative to } C \text { at } x .
\end{aligned}
$$

Recall from [21] that the inequality system $f \leq 0$ satisfies the weak BCQ at $x \in S_{f} \backslash \operatorname{int} S_{f}$ if

$$
N_{S_{f}}(x) \subseteq \text { cone } \partial f(x)+N_{\operatorname{dom} f}(x),
$$

where $S_{f}:=\{x \in X: f(x) \leq 0\}$. The following proposition describes a relationship between the BCQ and the weak BCQ .

Proposition 3.3. Let $f$ be a proper convex function on $X$ and $x \in S_{f} \backslash \operatorname{int} S_{f}$. Then the family $\left\{f, \delta_{\operatorname{dom} f}\right\}$ satisfies the $B C Q$ at $x$ if and only if the inequality system $f \leq 0$ satisfies the weak BCQ at $x$.

Proof. Write $g_{1}=f$ and $g_{2}=\delta_{\operatorname{dom} f}$. If $x$ satisfies, in addition, that $f(x)<0$, then both the necessary condition and the sufficient condition in the statement of the proposition are satisfied. In fact, since $x \notin \operatorname{int} S_{f},[21$, Lemma 2.2] states that

$$
N_{S_{f}}(x)=N_{\operatorname{dom} f}(x) .
$$

Consequently (3.11) holds, and the family $\left\{f, \delta_{\operatorname{dom} f}\right\}$ satisfies the BCQ at $x$ because of $(2.3)$ and $I(x)=$ $\{2\}($ as $f(x)<0)$ 
Therefore, to complete our proof we need only consider the case when $f(x)=0$. For this case note that $I(x)=\{1,2\}$. Thus, by (2.3), the family $\left\{f, \delta_{\operatorname{dom} f}\right\}$ satisfies the BCQ at $x$ if and only if

$$
N_{S_{f}}(x)=\operatorname{cone} \partial f(x)+N_{\operatorname{dom} f}(x) .
$$

Since the inclusion $N_{S_{f}}(x) \supseteq$ cone $\partial f(x)+N_{\operatorname{dom} f}(x)$ holds trivially (thanks to $f(x)=0$ and Remark 3.2(ii) as applied to $\left.\left\{f, \delta_{\operatorname{dom} f}\right\}\right),(3.11)$ and (3.12) are equivalent. This completes the proof.

\section{The Epigraph Hull Property}

Recall that the meaning of $\left\{g_{i}: i \in I\right\}, G, X, S$ and $I$ has been specified in section 2. The sup-function $G$ is sometimes denoted by $\sup _{i \in I} g_{i}$. Recall also that we always assume that $G$ is proper.

Definition 4.1. The family $\left\{g_{i}: i \in I\right\}$ is said to have

(i) the convex epigraph hull property (the convex EHP) if

$$
\operatorname{epi}\left(\sup _{i \in I} g_{i}\right)^{*}=\operatorname{co} \bigcup_{i \in I} \operatorname{epi} g_{i}^{*}
$$

(ii) the conical epigraph hull property (the conical EHP) if

$$
\text { epi } \sigma_{S}=\text { cone } \bigcup_{i \in I} \text { epi } g_{i}^{*} \text {. }
$$

Remark 4.1. It is routine to show that

$$
\operatorname{epi}\left(\sup _{i \in I} g_{i}\right)^{*} \supseteq \operatorname{co}_{i \in I} \text { epi } g_{i}^{*} \text { and } \quad \text { epi } \sigma_{S} \supseteq \operatorname{cone} \bigcup_{i \in I} \text { epi } g_{i}^{*} .
$$

Thus the family has the convex EHP (resp. conical EHP) if and only if

$$
\operatorname{epi}\left(\sup _{i \in I} g_{i}\right)^{*} \subseteq \operatorname{co} \bigcup_{i \in I} \text { epi } g_{i}^{*}, \quad\left(\text { resp. } \quad \text { epi } \sigma_{S} \subseteq \operatorname{cone} \bigcup_{i \in I} \text { epi } g_{i}^{*} .\right)
$$

Results in the following proposition are known: for (i) see [35] and for (ii) see [16,23,30]. Recall that we have assumed that $\sup _{i \in I} g_{i}$ is proper.

Proposition 4.1. Suppose in addition that each $g_{i}$ is lower semicontinuous. Then the following assertions regarding epigraphs hold:

(i)

$$
\operatorname{epi}\left(\sup _{i \in I} g_{i}\right)^{*}=\overline{\operatorname{co} \bigcup_{i \in I} \operatorname{epi} g_{i}^{*}} w^{*}
$$

(ii)

$$
\text { epi } \sigma_{S}=\operatorname{epi} \delta_{S}^{*}=\overline{\text { cone } \bigcup_{i \in I} \operatorname{epi} g_{i}^{*}} w^{*} \quad \text { if } S \text { is nonempty. }
$$

Corollary 4.1. Let $\left\{g_{i}: i \in I\right\}$ be as in the preceding proposition. Then the following assertions are valid:

(i) $\left\{g_{i}: i \in I\right\}$ has the convex EHP if and only if

$$
\text { co } \bigcup_{i \in I} \operatorname{epi} g_{i}^{*} \text { is } w^{*} \text {-closed; }
$$


(ii) $\left\{g_{i}: i \in I\right\}$ has the conical EHP if and only if

$$
\text { cone } \bigcup_{i \in I} \text { epi } g_{i}^{*} \text { is } w^{*} \text {-closed, }
$$

provided that $S \neq \emptyset$.

Proof. Since $G=\sup _{i \in I} g_{i}$ is proper, (4.3) holds. Thus (i) is seen to hold. Similarly, (ii) holds by (4.4) provided that $S \neq \emptyset$.

Remark 4.2. In [16, Definition 1] and [17, Definition 3.1] (under the assumption that each $g_{i}$ is lower semicontinuous), the system $\left\{\delta_{C} ; g_{i}, i \in I\right\}$ is said to be Farkas-Minkowski if

$$
\text { epi } \sigma_{C}+\text { cone } \bigcup_{i \in I} \text { epi } g_{i}^{*} \text { is } w^{*} \text {-closed. }
$$

Letting $i_{0} \notin I$ and writing $g_{i_{0}}:=\delta_{C}$, one sees that $\left\{\delta_{C} ; g_{i}, i \in I\right\}$ being Farkas-Minkowski is equivalent to $\left\{g_{i}, i \in I \cup\left\{i_{0}\right\}\right\}$ having conical EHP.

The following example shows that the lower semicontinuity assumption for $\left\{g_{i}: i \in I\right\}$ cannot be dropped in Proposition 4.1. In other words, Corollary 4.1 fails without lower semicontinity assumption on $\left\{g_{i}: i \in I\right\}$.

Example 4.1. Consider the real Hilbert space $l^{2}$ of square-summable series and let $\Omega_{+}$be the convex subset defined by

$$
\Omega_{+}:=\left\{x \in l^{2}: x_{i} \geq 0, \forall i \in \mathbb{N}, x_{i} \neq 0 \text { for at most finitely many } i\right\},
$$

where $x_{i}$ denotes the $i^{\text {th }}$ coordinate of $x$. Let $I=\{t \in \mathbb{R}: t>0\}$ and define a family $\left\{g_{t}: t \in I\right\}$ of proper convex functions by

$$
g_{t}(x):=\left\{\begin{array}{ll}
-t \sum_{i=1}^{\infty} i x_{i} & \text { if } x \in \Omega_{+} \\
+\infty & \text { otherwise }
\end{array} \quad \text { for each } t \in I .\right.
$$

Note in particular that $g_{t}(\cdot) \leq 0$ on $\operatorname{dom} g_{t}$, $\operatorname{dom} g_{t}=\Omega_{+}$, and $\left\{x: g_{t}(x) \leq 0\right\}=\Omega_{+}$for each $t \in I$. Thus $S:=\bigcap_{t \in I}\left\{x: g_{t}(x) \leq 0\right\}=\Omega_{+}$.

Let $y:=\left(1, \frac{1}{2}, \cdots, \frac{1}{n}, \cdots\right)$ and $y_{n}:=\left(1, \frac{1}{2}, \cdots, \frac{1}{n}, 0, \cdots\right)$ for each natural number $n$. Then $y \in l^{2} \backslash \Omega_{+}$ and $y_{n} \in \Omega_{+}$for each $n$. Furthermore, one has that $y_{n} \rightarrow y$ and

$$
\lim g_{t}\left(y_{n}\right)=\lim _{n \rightarrow \infty}-n t=-\infty<g_{t}(y)=+\infty \quad \text { for each } t \in I
$$

(so each $g_{t}$ is not lower semi-continuous); consequently, $\overline{g_{t}}(y)=-\infty$, where $\overline{g_{t}}$ denotes the closure of the function $g_{t}$ (cf. [40, Page 62]). Since $g_{t}^{*}={\overline{g_{t}}}^{*}$ by [40, Theorem 2.3.1(iv)], we see that

$$
g_{t}^{*}\left(x^{*}\right)=\bar{g}_{t}^{*}\left(x^{*}\right) \geq\left\langle x^{*}, y\right\rangle-\bar{g}_{t}(y)=+\infty \quad \text { for each } x^{*} \in l^{2} \text { and } t \in I .
$$

Thus

$$
\text { epi } g_{t}^{*}=\emptyset \quad \text { for each } t \in I \text {. }
$$

On the other hand, $\left(\sup _{t \in I} g_{t}\right)^{*}=\sigma_{\Omega_{+}}$which is proper since $\Omega_{+} \neq \emptyset$. Hence the lower semicontinuity assumption in Proposition 4.1 cannot be dropped.

Proposition 4.2. The family $\left\{g_{i}, \delta_{\mathrm{dom} g_{i}}: i \in I\right\}$ has the conical EHP if and only if the family $\left\{t g_{i}, \delta_{\text {dom } g_{i}}: i \in I, t>0\right\}$ has the convex EHP. 
Proof. By definition, the family $\left\{g_{i}, \delta_{\mathrm{dom} g_{i}}: i \in I\right\}$ has the conical EHP if and only if

$$
\text { epi } \sigma_{S}=\text { cone } \bigcup_{i \in I}\left(\text { epi } g_{i}^{*} \cup \text { epi } \sigma_{\text {dom } g_{i}}\right) \text {, }
$$

while the family $\left\{t g_{i}, \delta_{\text {dom } g_{i}}: i \in I, t>0\right\}$ has the convex EHP if and only if

$$
\operatorname{epi} \sigma_{S}=\operatorname{co} \bigcup_{i \in I}\left[\bigcup_{t>0} \operatorname{epi}\left(t g_{i}\right)^{*} \cup \operatorname{epi} \sigma_{\operatorname{dom} g_{i}}\right] \text {, }
$$

thanks to the easily checked equality $\delta_{S}=\sup _{t>0, i \in I} t g_{i}$. It suffices to prove that the sets on the right hand side of (4.5) and of (4.6) are equal. To do this, recall from [40, Theorem 2.3.1 (v)] that $(t G)^{*}\left(x^{*}\right)=t G^{*}\left(\frac{x^{*}}{t}\right)$ for each $t>0$ and $x^{*} \in X^{*}$. It follows that

$$
\text { epi }\left(t g_{i}\right)^{*}=t \text { epi } g_{i}^{*} \quad \text { for each } i \in I \text { and } t>0 \text {. }
$$

Since epi $\sigma_{\text {dom } g_{i}}$ is a cone, it follows that

$$
\begin{aligned}
\operatorname{co}_{i \in I}\left[\bigcup_{t>0} \operatorname{epi}\left(t g_{i}\right)^{*} \cup \text { epi } \sigma_{\operatorname{dom} g_{i}}\right] & =\operatorname{co~} \bigcup_{i \in I}\left[\bigcup_{t>0} t \text { epi } g_{i}^{*} \cup \text { epi } \sigma_{\operatorname{dom} g_{i}}\right] \\
& =\operatorname{co~} \bigcup_{i \in I} t\left(\operatorname{epi} g_{i}^{*} \cup \text { epi } \sigma_{\operatorname{dom} g_{i}}\right) \\
& =\operatorname{co} \bigcup_{i \in I} t\left(\operatorname{epi} g_{i}^{*} \cup \text { epi } \sigma_{\operatorname{dom} g_{i}}\right) \\
& =\operatorname{cone} \bigcup_{i \in I}\left(\operatorname{epi} g_{i}^{*} \cup \operatorname{epi} \sigma_{\operatorname{dom} g_{i}}\right)
\end{aligned}
$$

where the third equality holds because $(0,0) \in \operatorname{epi} \sigma_{\operatorname{dom} g_{i}}$ for each $i \in I$. This completes the proof.

Remark 4.3. The first part of the second conclusion of the following theorem was also independently obtained in [16, Corollary 2] for the special case when $g_{i}$ were assumed to be lower semicontinuous.

Theorem 4.1. The following assertions are valid:

(i) If the family $\left\{g_{i}: i \in I\right\}$ has the convex EHP, then it has the PLV property. The converse implication also holds if $\operatorname{dom} G^{*} \subseteq \operatorname{im} \partial G$.

(ii) Suppose $S \neq \emptyset$. If the family $\left\{g_{i}: i \in I\right\}$ has the conical EHP, then it satisfies the BCQ. The converse implication also holds if $\operatorname{dom} \sigma_{S} \subseteq \operatorname{im} \partial \delta_{S}$.

Proof. (i) Suppose that the family $\left\{g_{i}: i \in I\right\}$ has the convex EHP. By Remark 3.2(i), it suffices to show that (3.6) holds for each $x \in \operatorname{dom} \partial G$. Take $x^{*} \in \partial G(x)$. By $(2.2),\left(x^{*},\left\langle x^{*}, x\right\rangle-G(x)\right) \in \operatorname{epi} G^{*}$. Now (4.1) implies that $\left(x^{*},\left\langle x^{*}, x\right\rangle-G(x)\right)$ can be represented as

$$
\left(x^{*},\left\langle x^{*}, x\right\rangle-G(x)\right)=\sum_{i \in J} \lambda_{i}\left(x_{i}^{*}, \alpha_{i}\right)
$$

for some finite subset $J \subseteq I,\left(x_{i}^{*}, \alpha_{i}\right) \in \operatorname{epi} g_{i}^{*}, i \in J$ and $0<\lambda_{i} \leq 1$ with $\sum_{i \in J} \lambda_{i}=1$. This implies

$$
\left\langle x^{*}, x\right\rangle-G(x)=\sum_{i \in J} \lambda_{i} \alpha_{i} \geq \sum_{i \in J} \lambda_{i} g_{i}^{*}\left(x_{i}^{*}\right) \geq \sum_{i \in J} \lambda_{i}\left(\left\langle x_{i}^{*}, x\right\rangle-g_{i}(x)\right) .
$$

The equalities hold throughout (4.7) because $\left\langle x^{*}, x\right\rangle=\sum_{i \in J} \lambda_{i}\left\langle x_{i}^{*}, x\right\rangle$ and $g_{i}(x) \leq G(x)$ for each $i$. As $\lambda_{i} \neq 0$ for each $i \in J$, it follows that $g_{i}(x)=G(x)$ and $g_{i}^{*}\left(x_{i}^{*}\right)=\left\langle x_{i}^{*}, x\right\rangle-g_{i}(x)$ for each $i \in J$. Thus $J \subseteq \tilde{I}(x)$ and $x_{i}^{*} \in \partial g_{i}(x)$ for $i \in J$, thanks to (2.1). Hence

$$
x^{*}=\sum_{i \in \tilde{I}(x)} \lambda_{i} x_{i}^{*} \in \operatorname{co} \bigcup_{i \in \tilde{I}(x)} \partial g_{i}(x),
$$


i.e. the family $\left\{g_{i}: i \in I\right\}$ has the PLV property. This proves the first part of (i).

Now we assume $\operatorname{dom} G^{*} \subseteq \operatorname{im} \partial G$ and prove the converse implication. In view of Remark 4.1, we only need to show that

$$
\text { epi } G^{*} \subseteq \operatorname{co} \bigcup_{i \in I} \operatorname{epi} g_{i}^{*} \text {. }
$$

Take $\left(y^{*}, \alpha\right) \in \operatorname{epi} G^{*}$. Then $y^{*} \in \operatorname{dom} G^{*}$ and by assumption there exists $x \in X$ such that $y^{*} \in \partial G(x)$. Now (3.1) implies that $y^{*}$ can be represented as

$$
y^{*}=\sum_{i \in J} \lambda_{i} y_{i}^{*}
$$

for some finite subset $J \subseteq \tilde{I}(x), y_{i}^{*} \in \partial g_{i}(x)$ for each $i \in J$ and $0<\lambda_{i} \leq 1$ with $\sum_{i \in J} \lambda_{i}=1$. Note that, for each $i \in J,\left\langle y_{i}^{*}, x\right\rangle-G(x)=g_{i}^{*}\left(y_{i}^{*}\right)$ because $y_{i}^{*} \in \partial g_{i}(x)$ and $G(x)=g_{i}(x)$. Since

$$
\alpha \geq\left\langle y^{*}, x\right\rangle-G(x)=\sum_{i \in J} \lambda_{i}\left(\left\langle y_{i}^{*}, x\right\rangle-g_{i}(x)\right),
$$

there exists a set $\left\{\alpha_{i}: i \in J\right\}$ of real numbers such that

$$
\alpha=\sum_{i \in J} \lambda_{i} \alpha_{i} \quad \text { and } \quad g_{i}^{*}\left(y_{i}^{*}\right)=\left\langle y_{i}^{*}, x\right\rangle-g_{i}(x) \leq \alpha_{i} \quad \text { for each } j \in J .
$$

This implies that $\left(y_{i}^{*}, \alpha_{i}\right) \in \operatorname{epi} g_{i}^{*}$ for each $i$ and thus $\left(y^{*}, \alpha\right) \in \operatorname{co} \bigcup_{i \in I}$ epi $g_{i}^{*}$. Hence (4.8) is proved.

(ii) Suppose that the family has the conical EHP. We wish to show that it satisfies the BCQ, that is, to show that (3.8) holds for each $x \in S \backslash$ int $S$ (see Remark 3.2(iii)). Take $x \in S \backslash$ int $S$ and $x^{*} \in N_{S}(x)$. Since the set on the right hand side of (3.8) contains the origin, we assume without loss of generality that $x^{*} \neq 0$. By $(2.4),\left(x^{*},\left\langle x^{*}, x\right\rangle\right) \in$ epi $\sigma_{S}$. Now (4.2) implies that $\left(x^{*},\left\langle x^{*}, x\right\rangle\right)$ can be represented as

$$
\left(x^{*},\left\langle x^{*}, x\right\rangle\right)=\sum_{i \in J} \lambda_{i}\left(x_{i}^{*}, \alpha_{i}\right),
$$

for some finite subset $J \subseteq I,\left(x_{i}^{*}, \alpha_{i}\right) \in \operatorname{epi} g_{i}^{*}, \lambda_{i}>0, i \in J$. Then we have

$$
\left\langle x^{*}, x\right\rangle=\sum_{i \in J} \lambda_{i} \alpha_{i} \geq \sum_{i \in J} \lambda_{i} g_{i}^{*}\left(x_{i}^{*}\right) \geq \sum_{i \in J} \lambda_{i}\left(\left\langle x_{i}^{*}, x\right\rangle-g_{i}(x)\right) .
$$

Since $\left\langle x^{*}, x\right\rangle=\sum_{i \in J} \lambda_{i}\left\langle x_{i}^{*}, x\right\rangle$ and $g_{i}(x) \leq 0$ for each $i \in J$, the equalities in (4.9) hold throughout. Since $\lambda_{i} \neq 0$ for each $i \in J$, we obtain that for each $i \in J$

$$
g_{i}(x)=0,
$$

and

$$
g_{i}^{*}\left(x_{i}^{*}\right)=\left\langle x_{i}^{*}, x\right\rangle .
$$

It follows from (4.10) that $J \subseteq I(x)$. Also, summing up (4.10) and (4.11), we obtain for each $i \in J$ that

$$
g_{i}^{*}\left(x_{i}^{*}\right)+g_{i}(x)=\left\langle x_{i}^{*}, x\right\rangle,
$$

which, by (2.1), is equivalent to $x_{i}^{*} \in \partial g_{i}(x)$. Thus we have

$$
x^{*}=\sum_{i \in J} \lambda_{i} x_{i}^{*} \in \text { cone } \bigcup_{i \in I(x)} \partial g_{i}(x) .
$$

Therefore the family $\left\{g_{i}: i \in I\right\}$ satisfies the BCQ.

We now turn to the converse implication. Assume $\operatorname{dom} \sigma_{S} \subseteq \operatorname{im} \partial \delta_{S}$. In view of Remark 4.1, we only need to show that,

$$
\text { epi } \sigma_{S} \subseteq \text { cone } \bigcup_{i \in I} \text { epi } g_{i}^{*} \text {. }
$$


Take $\left(y^{*}, \alpha\right) \in$ epi $\sigma_{S}$. Since $(0,0)$ clearly belongs to the right hand side of (4.12), we assume without loss of generality that $\left(y^{*}, \alpha\right) \neq(0,0)$. Now, since $y^{*} \in \operatorname{dom} \sigma_{S} \subseteq \operatorname{im} \partial \delta_{S}$, there exists $x_{0} \in S$ such that $y^{*} \in \partial \delta_{S}\left(x_{0}\right)=N_{S}\left(x_{0}\right)$ by (2.3). The definition of BCQ implies that $y^{*}$ can be expressed as

$$
y^{*}=\sum_{i \in J} \lambda_{i} y_{i}^{*}
$$

for some finite subset $J \subseteq I\left(x_{0}\right), y_{i}^{*} \in \partial g_{i}\left(x_{0}\right)$ and $\lambda_{i} \geq 0$ for each $i \in J$. Note that, for each $i \in J$, $\left\langle y_{i}^{*}, x_{0}\right\rangle=g_{i}^{*}\left(y_{i}^{*}\right)$ because $y_{i}^{*} \in \partial g_{i}\left(x_{0}\right)$ and $g_{i}\left(x_{0}\right)=G\left(x_{0}\right)=0$. On the other hand, since $\alpha \geq\left\langle y^{*}, x_{0}\right\rangle=$ $\sum_{i \in J} \lambda_{i}\left\langle y_{i}^{*}, x_{0}\right\rangle$, there exists a set $\left\{\alpha_{i}: i \in J\right\}$ of real numbers such that

$$
\alpha=\sum_{i \in J} \lambda_{i} \alpha_{i} \quad \text { and } \quad g_{i}^{*}\left(y_{i}^{*}\right)=\left\langle y_{i}^{*}, x_{0}\right\rangle \leq \alpha_{i} \quad \text { for each } i \in J
$$

This implies that $\left(y_{i}^{*}, \alpha_{i}\right) \in \operatorname{epi} g_{i}^{*}$ for each $i$ and thus $\left(y^{*}, \alpha\right) \in$ cone $\bigcup_{i \in I}$ epi $g_{i}^{*}$. Hence (4.12) is proved.

Recall from [35] that a family of convex sets $\left\{C_{i}: i \in I\right\}$ in $X$ with nonempty intersection satisfies the sum of epigraphs constraint qualification (SECQ) if

$$
\text { epi } \sigma_{\bigcap_{i \in I} C_{i}}=\sum_{i \in I} \text { epi } \sigma_{C_{i}}
$$

The following proposition is on the relationships between the strong CHIP, the SECQ for a family of convex sets, and the conical EHP for the family consisting of the corresponding indicator functions.

Proposition 4.3. Let $\left\{C_{i}: i \in I\right\}$ be a family of convex sets in $X$ with nonempty intersection. Then the following assertions are valid:

(i) The family $\left\{C_{i}: i \in I\right\}$ has the strong CHIP if and only if the family of functions $\left\{\delta_{C_{i}}: i \in I\right\}$ has the $B C Q$;

(ii) The family $\left\{C_{i}: i \in I\right\}$ satisfies the SECQ if and only if the family of functions $\left\{\delta_{C_{i}}: i \in I\right\}$ has the conical EHP.

Proof. Consider the family of functions $\left\{\delta_{C_{i}}: i \in I\right\}$, i.e. $g_{i}:=\delta_{C_{i}}$ for each $i \in I$. Then,

$$
S:=\left\{x: \sup _{i \in I} \delta_{C_{i}}(x) \leq 0\right\}=\left\{x: \delta_{\bigcap_{i \in I} C_{i}}(x) \leq 0\right\}=\bigcap_{i \in I} C_{i} \neq \emptyset .
$$

Also, $G(x):=\sup _{i \in I} \delta_{C_{i}}(x)=\delta_{\bigcap_{i \in I} C_{i}}(x)$ and that $G(x)=0$ for each $x \in S$. Moreover, since $\delta_{C_{i}}(x)=0$ for each $x \in S$ and each $i \in I$, it follows that $I(x)=I$ for each $x \in S$.

(i) For each $x \in S$, we have from (2.3) that

$$
\text { cone } \bigcup_{i \in I(x)} \partial \delta_{C_{i}}(x)=\text { cone } \bigcup_{i \in I} \partial \delta_{C_{i}}(x)=\text { cone } \bigcup_{i \in I} N_{C_{i}}(x)=\sum_{i \in I} \operatorname{cone} N_{C_{i}}(x)=\sum_{i \in I} N_{C_{i}}(x) \text {. }
$$

Therefore, when $\left\{\delta_{C_{i}}: i \in I\right\}$ replaces $\left\{g_{i}: i \in I\right\}$, we see that (3.2) and (3.10) are equivalent and so (i) is proved.

(ii) Note that each epi $\sigma_{C_{i}}$ is a cone and so

$$
\text { cone } \bigcup_{i \in I} \text { epi } \sigma_{C_{i}}=\sum_{i \in I} \operatorname{cone}\left(\operatorname{epi} \sigma_{C_{i}}\right)=\sum_{i \in I} \text { epi } \sigma_{C_{i}} \text {. }
$$

Therefore, when $\left\{\delta_{C_{i}}: i \in I\right\}$ replaces $\left\{g_{i}: i \in I\right\}$, we see that (4.2) and (4.13) are equivalent and so (ii) is proved. 
Corollary 4.2 (see [35]). Let $\left\{C_{i}: i \in I\right\}$ be a family of convex sets in $X$ with nonempty intersection. If the family satisfies the SECQ, then it has the strong CHIP. The converse implication also holds if $\operatorname{dom} \sigma_{S} \subseteq \operatorname{im} \partial \delta_{S}$, where $S=\bigcap_{i \in I} C_{i}$.

Proof. The corollary follows from Proposition 4.3 and Theorem 4.1(ii) (applied to the family of functions $\left\{\delta_{C_{i}}: i \in I\right\}$ in place of $\left.\left\{g_{i}: i \in I\right\}\right)$.

Adopting a definition given in [36, Definition 5.3] originally in a more restrictive case, we say that a linear inequality

$$
\left\langle a^{*}, x\right\rangle \leq b
$$

(where $a^{*} \in X^{*}$ and $b \in \mathbb{R}$ ) is a consequence relation of (1.1) if every $x \in S$ satisfies (4.14). Moreover, the system (1.1) is said to be a convex Farkas-Minkowski (FM) system if every linear consequence relation of the system (1.1) is also a consequence relation of some finite subsystem of it. The following result was independently obtained in [16, Proposition 1] under the additional assumption that each $g_{i}$ is lower semicontinuous.

Proposition 4.4. Suppose that $S \neq \emptyset$ and that the family $\left\{g_{i}: i \in I\right\}$ has the conical EHP. Then the system (1.1) is a convex FM system.

Proof. Let $a^{*} \in X^{*}$ and $b \in \mathbb{R}$ be such that $\left\langle a^{*}, x\right\rangle \leq b$ for each $x \in S$. This means that

$$
\left(a^{*}, b\right) \in \operatorname{epi} \sigma_{S}
$$

thanks to (2.5). Since the family $\left\{g_{i}: i \in I\right\}$ has the conical EHP, it follows from (4.2) that

$$
\left(a^{*}, b\right) \in \text { cone } \bigcup_{i \in I} \text { epi } g_{i}^{*} .
$$

Thus, there exists a finite subset $J \subseteq I$ such that

$$
\left(a^{*}, b\right) \in \text { cone } \bigcup_{i \in J} \operatorname{epi} g_{i}^{*} \subseteq \operatorname{epi} \sigma_{S_{J}}
$$

where $S_{J}:=\left\{x \in X: g_{i}(x) \leq 0, \forall i \in J\right\}$ and the inclusion follows from Remark 4.1. Again by (2.5), one has $\left\langle a^{*}, x\right\rangle \leq b$ for each $x \in S_{J}$. This completes the proof.

The following corollary was proved by Li, Nahak and Singer in [36, Proposition 5.4] under the additional assumptions that $X=\mathbb{R}^{n}, S$ is compact and each $g_{i}$ is continuous (recall that if $S$ is a weakly compact convex set and $X$ is a normed linear space, then $\operatorname{dom} \sigma_{S} \subseteq \operatorname{im} \partial \delta_{S}$; see [35, Proposition 3.1]). A similar result was obtained in [16, Proposition 3], in which they assumed the family of lower semicontinuous functions to have BCQ at a point $z$ and deduced that every linear consequence relation (4.14) of the system (1.1) with $b=\left\langle a^{*} z\right\rangle$ is a consequence relation of some finite subsystem of it.

Corollary 4.3. Suppose that $S \neq \emptyset$ and that the family $\left\{g_{i}: i \in I\right\}$ satisfies the BCQ. Suppose further that $\operatorname{dom} \sigma_{S} \subseteq \operatorname{im} \partial \delta_{S}$. Then (1.1) is a convex FM system.

Proof. By Theorem 4.1(ii), the assumptions imply that the family $\left\{g_{i}: i \in I\right\}$ has the conical EHP. Hence the conclusion follows from Proposition 4.4.

\section{Optimality Conditions}

Let $X$ be a locally convex Hausdorff topological vector space as before. We use $\Gamma(X)$ to denote the class of all proper convex lower semicontinuous functions on $X$ as in [40]. For a subset of $X$, we define

$$
\mathcal{F}_{A}:=\left\{f \in \Gamma(X): \operatorname{dom} f \cap A \neq \emptyset \text {, epi } \sigma_{A}+\text { epi } f^{*} \text { is } w^{*} \text {-closed }\right\} .
$$


Since epi $\sigma_{A}=\operatorname{epi} \sigma_{\bar{A}}$ for any convex set $A$,

$$
f \in \mathcal{F}_{A} \Leftrightarrow f \in \mathcal{F}_{\bar{A}}
$$

It is known from [6, Theorem 3.2] that if $f \in \mathcal{F}_{A}$ and closed convex set $A$ are such that epi $\sigma_{A}+$ epi $f^{*}$ is $w^{*}$-closed, then the subdifferential sum formula holds:

$$
f \in \mathcal{F}_{A} \Rightarrow \partial\left(f+\delta_{A}\right)(x)=\partial f(x)+\partial \delta_{A}(x) \text { for each } x \in A \cap \operatorname{dom} f
$$

Thus, (5.1) entails that

$$
f \in \mathcal{F}_{A} \Rightarrow \partial\left(f+\delta_{\bar{A}}\right)(x)=\partial f(x)+\partial \delta_{\bar{A}}(x) \quad \text { for each } x \in \bar{A} \cap \operatorname{dom} f
$$

As in [40], let $\Lambda(X)$ denote the class of all proper convex functions on $X$. Let $f \in \Lambda(X)$, and recall that the meaning of $\left\{g_{i}: i \in I\right\}, G, X, S$ and $I$ has been specified in section 2 and that we always assume that $G$ is proper. We consider the following minimization problem:

$$
\begin{array}{ll}
\text { Minimize } & f(x), \\
\text { s. t. } & g_{i}(x) \leq 0, i \in I .
\end{array}
$$

Clearly, $\bar{x} \in S$ is a minimizer of (5.3) if and only if it is a minimizer of (5.4) defined as follows:

$$
\begin{array}{ll}
\text { Minimize } & f(x), \\
\text { s. t. } & x \in S .
\end{array}
$$

The following theorem gives a characterization for a feasible point $\bar{x}$ to be a minimizer. Note in particular that it improves a result in [16, Theorem 4] as far as the lower semicontinuity of the functions $g_{i}$ is relaxed. See also [5] for other related results. For $h \in \Lambda(X)$, let cont $h$ denote the set of all points at each of which $h$ is continuous, that is

$$
\text { cont } h:=\{x \in X: h \text { is continuous at } x\} .
$$

Theorem 5.1. Let $\bar{x}$ be a feasible point of (5.3). Then the following statements are equivalent:

(i) The family $\left\{g_{i}: i \in I\right\}$ satisfies the BCQ at $\bar{x}$.

(ii) For each $f \in \mathcal{F}_{S}, \bar{x}$ is a minimizer of (5.4) with $\bar{S}$ in place of $S$ if and only if there exist a finite subset $J \subseteq I(\bar{x})$ and $\lambda_{i} \geq 0, i \in J$ such that

$$
0 \in \partial f(\bar{x})+\sum_{i \in J} \lambda_{i} \partial g_{i}(\bar{x}) .
$$

(iii) For any $f \in \Lambda(X)$ such that cont $f \cap S \neq \emptyset, \bar{x}$ is a minimizer of (5.4) if and only if there exist a finite subset $J \subseteq I(\bar{x})$ and $\lambda_{i} \geq 0, i \in J$ such that (5.5) holds.

(iv) For each continuous linear functional $f, \bar{x}$ is a minimizer of (5.4) if and only if there exist a finite subset $J \subseteq I(\bar{x})$ and $\lambda_{i} \geq 0, i \in J$ such that (5.5) holds.

Proof. Recall a well known result in convex analysis (cf. [40, Theorem 2.5.7]) that if $f \in \Lambda(X)$ and $A$ is a convex subset, then

$$
\bar{x} \text { minimizes } f \text { on } A \Longleftrightarrow \bar{x} \text { minimizes }\left(f+\delta_{A}\right) \text { on } X \Longleftrightarrow 0 \in \partial\left(f+\delta_{A}\right)(\bar{x}) \text {. }
$$

We now first prove (i) $\Rightarrow$ (ii). Fix $f \in \mathcal{F}_{S}$. By $(5.2)$, we have

$$
\partial\left(f+\delta_{\bar{S}}\right)(x)=\partial f(x)+\partial \delta_{\bar{S}}(x) \quad \text { for each } x \in \bar{S} \cap \operatorname{dom} f .
$$


By (2.3), we know further that

$$
\partial \delta_{\bar{S}}(x)=N_{\bar{S}}(x)=N_{S}(x) \quad \text { for each } x \in S
$$

Thus by (5.6), (5.7) and (5.8), the assumption (i) implies the following equivalences:

$$
\bar{x} \text { minimizes } f \text { on } \bar{S} \Longleftrightarrow 0 \in \partial f(\bar{x})+N_{S}(\bar{x}) \Longleftrightarrow 0 \in \partial f(\bar{x})+\text { cone } \bigcup_{i \in I(\bar{x})} \partial g_{i}(\bar{x}) \text {. }
$$

The implication (i) $\Rightarrow$ (ii) is now clear.

Next, we prove (i) $\Rightarrow$ (iii). Let $f \in \Lambda(X)$ be such that cont $f \cap S \neq \emptyset$. Then [40, Theorem 2.8.7(iii)] states that

$$
\partial\left(f+\delta_{S}\right)(x)=\partial f(x)+\partial \delta_{S}(x) \text { for each } x \in S \cap \operatorname{dom} f .
$$

Thus the implication (i) $\Rightarrow$ (iii) is seen to hold by (5.6) and (5.9).

To show the implication (ii) $\Rightarrow(\mathbf{i v})$, let $f$ be a continuous linear functional on $X$. Then inf $x \in S f(x)=$ $\inf _{x \in \bar{S}} f(x)$ and $f \in \mathcal{F}_{\bar{S}}$ by [15, Remark 5.6]. The latter condition is equivalent to $f \in \mathcal{F}_{S}$, thanks to (5.1). Since $\bar{x} \in S$, the implication (ii) $\Rightarrow$ (iv) is clear.

The implication (iii) $\Rightarrow$ (iv) is immediate.

Finally, we turn to the proof of (iv) $\Rightarrow(\mathbf{i})$. We need to show that $(3.8)$ holds for $x=\bar{x}$. Let $y^{*} \in N_{S}(\bar{x})$. Then $\bar{x}$ is a minimizer of the following optimization problem:

$$
\begin{array}{ll}
\text { Minimize } & -\left\langle y^{*}, x\right\rangle \\
\text { s. t. } & x \in S .
\end{array}
$$

By (iv), there exist a finite subset $J \subseteq I(\bar{x})$ and $\lambda_{i} \geq 0, i \in J$ such that

$$
0 \in-y^{*}+\sum_{i \in J} \lambda_{i} \partial g_{i}(\bar{x})
$$

Thus

$$
y^{*} \in \sum_{i \in J} \lambda_{i} \partial g_{i}(\bar{x}) \subseteq \text { cone } \bigcup_{i \in I(\bar{x})} \partial g_{i}(\bar{x}) .
$$

Therefore $\bar{x}$ satisfies (3.8) as required to show. This completes the proof.

Let $A$ be a convex set in $X$ and $a \in A$. In the literature there are several unequivalent conditions on "the relative interior" of $A$ such as

$$
a \in U \cap \overline{\operatorname{aff} A} \subseteq A
$$

and

$$
a \in U \cap \operatorname{aff} A \subseteq A
$$

where $U$ is a neighborhood of $a$. For example, (5.10) was considered in [3,20] and (5.11) in [2,40]. As (5.11) is not needed for our present study, we will follow the terminology of $[3,20]$ to say that a is in the relative interior of $A$ and denoted by $a \in$ ri $A$, if there exists a neighborhood of $a$ such that (5.10) holds.

Remark 5.1. If $0 \in \operatorname{ri} A$ and $x_{0} \in \bar{A}$, then $t_{0} \in \operatorname{ri} A$ for each $t \in[0,1)$. To see this, consider the Minkowski functional $p_{A}(x):=\inf \left\{\lambda: \lambda^{-1} x \in A\right\}$ for each $x \in \overline{\operatorname{span} A}$. By considering $A$ and $\bar{A}$ as subsets of $\overline{\operatorname{span} A}$, we have ri $A=\left\{x \in \overline{\operatorname{span} A}: p_{A}(x)<1\right\}$ and $\bar{A}=\left\{x \in \overline{\operatorname{span} A}: p_{A}(x) \leq 1\right\}$ (cf. [40, Proposition 1.1.1(ii)]). Since $p_{A}\left(x_{0}\right) \leq 1$, we have $p_{A}\left(t x_{0}\right)=t p_{A}\left(x_{0}\right)<1$ for each $t \in[0,1)$. Thus $t_{0} \in \operatorname{ri} A$ as required.

For $f \in \Lambda(X)$, define

$$
\operatorname{cont}_{A} f=\left\{x \in \operatorname{dom} f \cap \overline{\operatorname{aff} A}:\left.\quad f\right|_{\overline{a f f} A} \text { is continuous at } x\right\} .
$$


Remark 5.2. If $0 \in \operatorname{cont}_{A} f$ and $x_{0} \in \operatorname{dom} f \cap \overline{\operatorname{span} A}$, then $t_{0} \in \operatorname{cont}_{A} f$ for all $t \in[0,1)$. To see this, consider $\operatorname{dom} f \cap \overline{\operatorname{span} A}$ as a subset of $\overline{\operatorname{span} A}$; in particular, $0 \in \operatorname{cont}_{A} f \subseteq \operatorname{int}(\operatorname{dom} f \cap \overline{\operatorname{span}} A)$. Since $x_{0} \in \operatorname{dom} f \cap \overline{\operatorname{span} A}$, it follows that $x_{0} \in \operatorname{int}(\operatorname{dom} f \cap \overline{\operatorname{span}} A)$ for all $t \in[0,1)$. Consequently, by the convexity of $f, t x_{0} \in \operatorname{cont}_{A} f$ for all $t \in[0,1)$ thanks to [40, Theorem 2.2.9] because $\left.f\right|_{\overline{\operatorname{span} A}}$ is continuous at 0 .

Lemma 5.1. Consider the problem (5.4) and let $f \in \Lambda(X)$. Suppose that

$$
(\operatorname{dom} f \cap \operatorname{ri} S) \cup\left(\bar{S} \cap \operatorname{cont}_{S} f\right) \neq \emptyset .
$$

Then

$$
\inf _{x \in S} f(x)=\inf _{x \in \bar{S}} f(x) .
$$

Proof. By (5.12), we assume without loss of generality that

$$
0 \in(\operatorname{dom} f \cap \operatorname{ri} S) \cup\left(\bar{S} \cap \operatorname{cont}_{S} f\right) .
$$

Let $\lambda>\inf _{x \in \bar{S}} f(x)$ and take $x_{0} \in \bar{S}$ such that $\lambda>f\left(x_{0}\right)$. To show (5.13) it suffices to show that $\lambda>\inf _{x \in S} f(x)$. By the convexity we have

$$
f\left(t x_{0}\right) \leq t f\left(x_{0}\right)+(1-t) f(0) \quad \text { for each } t \in[0,1] .
$$

Letting $t \uparrow 1$ in (5.14), we obtain

$$
\limsup _{t \rightarrow 1^{-}} f\left(t x_{0}\right) \leq \lim _{t \rightarrow 1^{-}}\left[t f\left(x_{0}\right)+(1-t) f(0)\right]=f\left(x_{0}\right)<\lambda .
$$

This and Remark 5.1 imply that $\inf _{x \in S} f(x) \leq f\left(x_{0}\right)$ if $0 \in \operatorname{ri} S$ (so $t x_{0} \in \operatorname{ri} S$ for each $t \in[0,1)$ ). It remains to consider the case when $0 \in \bar{S} \cap \operatorname{cont}_{S} f$. But then Remark 5.2 entails that $t x_{0}$ is a continuity point of $\left.f\right|_{\overline{\operatorname{span} S}}$ if $t \in[0,1)$. Noting $t x_{0} \in \bar{S}$, it follows from (5.15) that $f\left(x_{t}\right)<\lambda$ for $x_{t} \in S$ close enough to $t x_{0}$, provided that $t<1$ sufficiently near to 1 . Therefore $\inf _{x \in \bar{S}} f(x)<\lambda$ in any case. This completes the proof.

Remark 5.3. For two convex sets $A, C$ in a Banach space $X$, recall from [34] that an $a \in A$ belongs to rint $_{\overline{\text { aff } C}} A$ if $a \in B(a, \epsilon) \cap \overline{\operatorname{aff} C} \subseteq A$ for some $\epsilon>0$. Note that if $X$ is a Banach space and $f \in \Gamma(X)$, then

$$
\operatorname{rint}_{\overline{\text { aff } S}} \operatorname{dom} f \subseteq \operatorname{cont}_{S} f .
$$

To see this we assume without loss of generality that $0 \in S$. Then $\overline{\operatorname{aff} S}=\overline{\operatorname{span} S}$ is a Banach space. Since $\left.f\right|_{\overline{\mathrm{aff} S}} \in \Gamma(\overline{\mathrm{aff}} \mathrm{S})$, (5.16) follows from [40, Theorem 2.2.20].

Corollary 5.1. Under the assumption of Theorem 5.1, for any $\bar{x} \in S$, the following statements are equivalent:

(i) The family $\left\{g_{i}: i \in I\right\}$ satisfies the $B C Q$ at $\bar{x}$.

(ii') For each $f \in \mathcal{F}_{S}$ satisfying (5.12), $\bar{x}$ is a minimizer of (5.4) if and only if there exist a finite subset $J \subseteq I(\bar{x})$ and $\lambda_{i} \geq 0, i \in J$ such that (5.5) holds.

Proof. Suppose (i) holds. Then Theorem 5.1(ii) holds. Let $f \in \mathcal{F}_{S}$ satisfy (5.12). Then $\inf _{x \in S} f(x)=$ $\inf _{x \in \bar{S}} f(x)$ by Lemma 5.1. Since $\bar{x} \in S$, applying Theorem 5.1(ii) to this $f$, (ii') is seen to hold. Conversely, suppose (ii') holds. Then part (iv) (and so part (i)) of Theorem 5.1 holds because any continuous linear functional $f$ on $X$ belongs to $\mathcal{F}_{S}$ (by [15, Remark 5.6] and (5.1)), and satisfies (5.12) (since $\operatorname{dom} f=X$ ). The proof is complete. 
The following result was proved in [15, Theorem 5.5] under the additional assumption that each $g_{i}$ is continuous, which was recently extended in [16, Theorem 3] to the setting that some $g_{i}$ are allowed merely lower semi-continuous.

Corollary 5.2. Suppose that $f \in \mathcal{F}_{S}$ and that the family $\left\{g_{i}: i \in I\right\}$ has the conical EHP. Assume that either $S$ is closed or the condition (5.12) is satisfied. Let $\bar{x} \in S$. Then $\bar{x}$ is a minimizer of (5.4) if and only if there exist a finite subset $J \subseteq I(\bar{x})$ and $\lambda_{i} \geq 0, i \in J$ such that

$$
0 \in \partial f(\bar{x})+\sum_{i \in J} \lambda_{i} \partial g_{i}(\bar{x})
$$

Proof. Since the family $\left\{g_{i}: i \in I\right\}$ has the conical EHP, it has the BCQ at $\bar{x}$ by Theorem 4.1(ii). Moreover (5.13) holds by the assumptions and Lemma 5.1. Since $\bar{x} \in S$ it follows that $\bar{x}$ is a minimizer of (5.4) if and only if $\bar{x}$ is a minimizer of (5.4) but with $\bar{S}$ in place of $S$. Thus the corollary follows from the implication (i) $\Rightarrow$ (ii) in Theorem 5.1.

The following example shows that (5.13) and the related corollaries may fail if the assumption (5.12) is dropped.

Example 5.1. Define $S:=\left\{(x, y) \in \mathbb{R}^{2}: x \geq 0\right\} \backslash\left\{(0, y) \in \mathbb{R}^{2}: y<1\right\}$ and

$$
f(x, y):= \begin{cases}\frac{y^{2}}{2} & \text { if } x=0 \\ +\infty & \text { otherwise }\end{cases}
$$

Then $f$ is proper convex lower semicontinuous, $S$ is convex and $\bar{S}=\left\{(x, y) \in \mathbb{R}^{2}: x \geq 0\right\}$. Note that in this case $\operatorname{dom} f=\left\{(0, y) \in \mathbb{R}^{2}: y \in \mathbb{R}\right\}$ which is disjoint from the set ri $S=\operatorname{int} S=\left\{(x, y) \in \mathbb{R}^{2}: x>0\right\}$. It is easy to see that $\inf _{(x, y) \in S} f(x, y)=f(0,1)=\frac{1}{2}$ but $\inf _{(x, y) \in \bar{S}} f(x, y)=f(0,0)=0$. Thus (5.13) fails.

Next we wish to show that $f \in \mathcal{F}_{S}$. To do this, note first that for each $(x, y) \in \mathbb{R}^{2}$

$$
f^{*}(x, y)=\sup \{\langle(u, v),(x, y)\rangle-f(u, v):(u, v) \in \operatorname{dom} f\}=\frac{y^{2}}{2},
$$

and that

$$
\sigma_{S}(x, y)=\sup _{u \geq 0, v \in \mathbb{R}}\langle(u, v),(x, y)\rangle= \begin{cases}0 & \text { if } x \leq 0 \text { and } y=0 \\ +\infty & \text { otherwise. }\end{cases}
$$

It is easy to see by the definition that

$$
\text { epi } f^{*}=\left\{(x, y, r) \in \mathbb{R}^{3}: \frac{y^{2}}{2} \leq r\right\} \quad \text { and } \quad \text { epi } \sigma_{S}=\left\{(x, 0, r) \in \mathbb{R}^{3}: x \leq 0, r \geq 0\right\} \text {. }
$$

Therefore, epi $f^{*}+$ epi $\sigma_{S}=$ epi $f^{*}$. This implies that epi $f^{*}+$ epi $\sigma_{S}$ is weak $k^{*}$-closed. Since $f$ is proper and $\operatorname{dom} f \cap S \neq \emptyset$, we see that $f \in \mathcal{F}_{S}$.

Now, note that $(0,1)$ is the minimizer of the following problem:

$$
\begin{array}{ll}
\text { Minimize } & f(x, y) \\
\text { s. t. } & \delta_{S}(x, y) \leq 0 .
\end{array}
$$

Note also that $\partial f(0,1)=\{(x, 1): x \in \mathbb{R}\}$ and that $N_{S}(0,1)=\{\lambda(-1,0): \lambda \geq 0\}$. Hence the optimality condition $(0,0) \in \partial f(0,1)+N_{S}(0,1)$ fails even though $f \in \mathcal{F}_{S}$ and the family $\left\{\delta_{S}\right\}$ has the BCQ property. 


\section{Applications to Conic Programming}

We continue our study of the conic programming problem with notations as explained in Example 2.1. It can be checked in a straightforward manner that the following facts are true. They are known when $g$ is continuous on $X$; see $[24,30]$. Some related results can be founded in [17].

Fact 6.1. cone $\bigcup_{\lambda \in K^{\oplus}} \operatorname{epi}(\lambda g)^{*}=\bigcup_{\lambda \in K^{\oplus}} \operatorname{epi}(\lambda g)^{*}$.

Fact 6.2. cone $\bigcup_{\lambda \in K^{\oplus}} \partial(\lambda g)(x)=\bigcup_{\lambda \in K^{\oplus}} \partial(\lambda g)(x)$ for each $x \in X$.

Fact 6.3. cone $\bigcup_{\substack{\lambda \in K^{\oplus} \\ \lambda g(x)=0}} \partial(\lambda g)(x)=\bigcup_{\substack{\lambda \in K^{\oplus} \\ \lambda g(x)=0}} \partial(\lambda g)(x)=N g(x)_{0}$ for each $x \in X$, where $N g(x)_{0}$ is defined by

$$
N g(x)_{0}:=\left\{u^{*} \in X^{*}:\left(u^{*}, u^{*}(x)\right) \in \bigcup_{\lambda \in K^{\oplus}} \operatorname{epi}(\lambda g)^{*}\right\} .
$$

Generalizing the corresponding notions in $[24,26,29]$ to suit our present noncontinuous situation we make the following definitions (it is a routine to see that the notions in the following definition coincide with corresponding ones in $[24,26,29]$ in the case when $g: X \rightarrow Z$ is continuous):

Definition 6.1. For $g, C, K$ as in Example 2.1, we say that

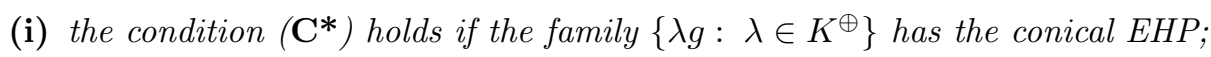

(ii) the closed cone constraint qualification (CCCQ) holds if the family $\left\{\delta_{C}, \lambda g: \lambda \in K^{\oplus}\right\}$ has the conical EHP;

(iii) the pair $\left\{C, g^{-1}(-K)\right\}$ has the sharpened strong CHIP at $x \in C \cap g^{-1}(-K)$ if $N_{C \cap g^{-1}(-K)}(x)=$ $N_{C}(x)+N g(x)_{0}$.

The following notion of $K$-lower semicontinuity was introduced in [37] and extended in $[1,12]$ for functions $g: X \rightarrow Z \bullet$. It was also considered in [4].

Definition 6.2. For $g, K$ as in Example 2.1, the function $g$ is said to be $K$-lower semicontinuous at $x_{0} \in X$ if for each neighborhood $V$ of zero in $Z$ and any $b \in Z$ with $b \leq_{K} g\left(x_{0}\right)$, there exists a neighborhood $U$ of zero in $X$ such that

$$
g\left(x_{0}+U\right) \subseteq b+V+K \cup\{\infty\} .
$$

Clearly, if $g: X \rightarrow Z$ is continuous, then $g$ is $K$-convex lower semicontinuous. Below we give an example of a function that is $K$-convex lower semicontinuous but not continuous.

Example 6.1. Let $X=l^{1}$ and $Z=l^{1}$ respectively under the $l^{\infty}$-norm $\|\cdot\|_{\infty}$ and the $l^{1}$-norm $\|\cdot\|_{1}$, and let $g$ denote the identity map from $X$ into $Z$. Then $g$ has the desired properties (that $g$ is not continuous is well-known). To see this, let us fix a nonzero element $c \in X$ and let $K$ denote its kernel in $Z$, that is $K:=\{z \in Z:\langle c, z\rangle=0\}$. By a well-known result (cf. [39, Page 24]), the distance to each $z \in Z$ from the closed subspace $K$ satisfies the so-called Ascoli formula:

$$
d(z, K)=\frac{|\langle c, z\rangle|}{\|c\|_{\infty}}, \text { for each } z \in Z
$$

and it follows that

$$
d(z, K) \leq \alpha\|z\|_{\infty}, \text { for each } z \in Z,
$$

where $\alpha:=\frac{\|c\|_{1}}{\|c\|_{\infty}}$; in particular we have

$$
d(g(x), K) \leq \alpha\|g(x)\|_{\infty}, \text { for each } x \in X,
$$

This implies that $g$ is $K$-lower semicontinuous at $x_{0}:=0$ (for $\epsilon>0$ and $V=\left\{z \in Z:\|z\|_{1}<\epsilon\right\}$, (6.1) holds with $\left.U=\left\{x \in X:\|x\|_{\infty}<\frac{\epsilon}{\alpha}\right\}\right)$. By the linearity of $g$, we conclude that $g$ is $K$-lower semicontinuous on the whole $X$. 
Proposition 6.1. Let $g, K$ be as in Example 2.1. Suppose that $g$ is $K$-lower semicontinuous and that $\operatorname{dom} g$ is closed. Then for each $\lambda \in K^{\oplus}, \lambda g$ is lower semicontinuous.

Proof. Let $\lambda \in K^{\oplus} \backslash\{0\}$ and let $x_{0} \in X$. To show the lower semicontinuity of $\lambda g$ at $x_{0}$, we assume without loss of generality that $x_{0} \in \operatorname{dom} g$ (thanks to the assumption that dom $g$ is closed). Let $\epsilon>0$. By the continuity of $\lambda$, take a neighborhood $V$ of zero in $Z$ such that $|\lambda(v)|<\epsilon$ for each $v \in V$. By definition of $K$-lower semicontiuity, there exists a neighbourhood $U$ of $x_{0}$ in X such that (6.1) holds. Let $u \in x_{0}+U$. By (6.1), there exist $v_{1} \in V$ and $k \in K \cup\{\infty\}$ such that $g(u)=g\left(x_{0}\right)+v_{1}+k$. For the case when $k \in K$, we have

$$
\lambda g\left(x_{0}\right)=\left\langle\lambda, g\left(x_{0}\right)\right\rangle \leq\left\langle\lambda, g(u)-v_{1}\right\rangle<\lambda g(u)+\epsilon .
$$

For the case when $k=\infty$, one has $g(u)=\infty$, i.e., $u \notin \operatorname{dom} g$. Thus, it follows from definition that $\lambda g\left(x_{0}\right)-\epsilon<\lambda g(u)=\infty$. Therefore, $\lambda g$ is lower semicontinuous at $x_{0}$.

Example 6.2 below shows that the converse of Proposition 6.1 is not true.

Example 6.2. Let $X=L^{2}[0,1], Z=L^{2}[0,1]$ respectively under $\|\cdot\|_{1}$-norm and $\|\cdot\|_{2}$-norm. Let $K=\{0\}$ and let $Z \bullet:=Z \cup\{+\infty\}$ as in Example 2.1. Let $D=\left\{x \in X:\|x\|_{2} \leq 1\right\}$ and let $g(x)=x+\delta_{D}(x)$. Then $g$ is not continuous on $D$ (as there exists a sequence $\left\{x_{n}\right\}$ in $X$ such that each $\left\|x_{n}\right\|_{2}=1$ but $\left\|x_{n}\right\|_{1}=\frac{1}{n}$; for example, let $x_{n}=n \chi_{\left[0,1 / n^{2}\right]}$ be the characteristic function of the interval $\left.\left[0,1 / n^{2}\right]\right)$, and so $g$ is not $\{0\}$-lower semicontinuous.

Let $\lambda \in Z^{*}=\{0\}^{\oplus}$. We claim that $\lambda g$ is lower semicontinuous. Let $r \in \mathbb{R}$ and let

$$
A_{r}:=\{x \in X: \lambda g(x) \leq r\} .
$$

It suffices to show that $A_{r}$ is closed in $X$. To do this, let $x \in X$ and $\left\{x_{n}\right\}$ be a sequence in $A_{r}$ such that $\left\|x_{n}-x\right\|_{1} \rightarrow 0$. By an elementary result in Lebesgue theory (see [38, Page 95, Proposition 18]), there exists a subsequence $\left\{x_{n_{k}}\right\}$ convergent to $x$ almost everywhere. By Fatou's lemma and the fact that $A_{r} \subseteq D$ (by (2.7)), it follows that

$$
\int_{0}^{1}|x|^{2} d t \leq \liminf _{k} \int_{0}^{1}\left|x_{n_{k}}\right|^{2} d t \leq 1
$$

thus $x \in D$. Let $\epsilon>0$. Since $\lambda \in L^{2}[0,1]$, there exists a simple function $h$ such that $\|\lambda-h\|_{2}<\epsilon$. Noting $\left\|x-x_{n}\right\|_{2} \leq 2$ (as $x, x_{n} \in D$ ), it follows from the Hölder inequality that

$$
\begin{aligned}
\lambda g(x)-\lambda g\left(x_{n}\right) & =\left\langle\lambda, x-x_{n}\right\rangle=\left\langle\lambda-h, x-x_{n}\right\rangle+\left\langle h, x-x_{n}\right\rangle \\
& \leq 2 \epsilon+\|h\|_{\infty}\left\|x-x_{n}\right\|_{1} \rightarrow 2 \epsilon .
\end{aligned}
$$

This implies that

$$
\lambda g(x) \leq \liminf _{n} \lambda g\left(x_{n}\right)+2 \epsilon \leq r+2 \epsilon,
$$

and hence that $\lambda g(x) \leq r$ as $\epsilon>0$ is arbitrary. Therefore $x \in A_{r}$ and $A_{r}$ is closed as wished to show.

By Fact 6.1, Corollary 4.1 and Proposition 6.1, the following remark is obvious:

Remark 6.1. Let $g, C, K$ be as in Example 2.1. Suppose in addition that $g$ is $K$-lower semicontinuous and that $\operatorname{dom} g$ is closed. Then the condition ( $\left.\mathbf{C}^{*}\right)$ holds if and only if

$$
\bigcup_{\lambda \in K^{\oplus}} \operatorname{epi}(\lambda g)^{*} \text { is } w^{*} \text {-closed; }
$$

more generally, the CCCQ holds if and only if

$$
\text { epi } \sigma_{C}+\bigcup_{\lambda \in K^{\oplus}} \operatorname{epi}(\lambda g)^{*} \text { is } w^{*} \text {-closed. }
$$

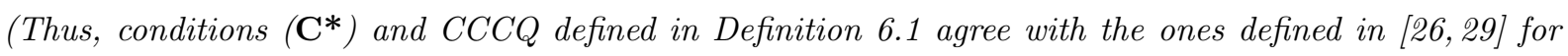
the continuous case.) 
Corollary 6.1. The following equivalence holds for any $x \in C \cap g^{-1}(-K)$ :

The family $\left\{\delta_{C}, \lambda g: \lambda \in K^{\oplus}\right\}$ satisfies the $B C Q$ at $x$ $\Leftrightarrow\left\{C, g^{-1}(-K)\right\}$ has the sharpened strong CHIP at $x$.

Proof. Note first that $\left\{x: \delta_{C}(x) \leq 0, \lambda g(x) \leq 0, \lambda \in K^{\oplus}\right\}=C \cap g^{-1}(-K)$. Hence the family $\left\{\delta_{C}, \lambda g\right.$ : $\left.\lambda \in K^{\oplus}\right\}$ satisfies the BCQ at $x$ if and only if

$$
N_{C \cap g^{-1}(-K)}(x)=N_{C}(x)+\text { cone } \bigcup_{\substack{\lambda \in K^{\oplus} \\ \lambda g(x)=0}} \partial(\lambda g)(x),
$$

which is equivalent to $\left\{C, g^{-1}(-K)\right\}$ has the sharpened strong CHIP at $x$, by Fact 6.3 .

The next two corollaries were respectively proved in [24, Propositions 3.3 and 3.4] under the additional assumption that $g$ is continuous.

Corollary 6.2. If $g, C, K$ are as in Example 2.1 and the CCCQ holds, then

$$
N_{C \cap g^{-1}(-K)}(x)=N_{C}(x)+\bigcup_{\substack{\lambda \in K^{\oplus} \\ \lambda g(x)=0}} \partial(\lambda g)(x), \text { for each } x \in C \cap g^{-1}(-K),
$$

that is, $\left\{C, g^{-1}(-K)\right\}$ has the sharpened strong CHIP at each point in $C \cap g^{-1}(-K)$. In particular, if the condition $\left(\mathbf{C}^{*}\right)$ holds, then

$$
N_{g^{-1}(-K)}(x)=\bigcup_{\substack{\lambda \in K^{\oplus} \\ \lambda g(x)=0}} \partial(\lambda g)(x), \text { for each } x \in g^{-1}(-K) .
$$

Proof. We need only prove the first assertion. Define $I:=K^{\oplus} \cup\left\{i_{0}\right\}, i_{0} \notin K^{\oplus}$, and consider $\left\{g_{i}: i \in I\right\}$ as defined in (2.8). Then $S:=\left\{x: g_{i}(x) \leq 0\right\}$ is exactly $C \cap g^{-1}(-K)$ and the active index set $I(x)$ is exactly $\left\{i_{0}\right\} \cup\left\{\lambda \in K^{\oplus}: \lambda g(x)=0\right\}$. Thus (6.2) simply means that the family $\left\{g_{i}: i \in I\right\}$ has the BCQ, and hence the result follows from Remark 6.1 and Theorem 4.1.

Corollary 6.3. If $g, C, K$ are as in Example 2.1 and the condition (C*) holds, then for each $x \in$ $C \cap g^{-1}(-K)$, the family $\left\{C, g^{-1}(-K)\right\}$ satisfies the strong CHIP at $x$ if and only if it satisfies the sharpened strong CHIP at $x$.

Proof. By the given assumption, (6.3) holds, that is, $N_{g^{-1}(-K)}(x)=N g(x)_{0}$ for each $x \in g^{-1}(-K)$. Consequently, the following equivalence holds for each $x \in C \cap g^{-1}(-K)$ :

$$
N_{C \cap g^{-1}(-K)}(x)=N_{C}(x)+N_{g^{-1}(-K)}(x) \Leftrightarrow N_{C \cap g^{-1}(-K)}(x)=N_{C}(x)+N g(x)_{0} .
$$

Thus the result is clear.

\section{Applications to Best Approximation Theory}

Let us recall from [14] that for a system of finitely many closed convex sets $\left\{D, C_{i}: i \in I\right\}$ in a Hilbert space, where $C_{i}=\left\{x \in X:\left\langle a_{i}, x\right\rangle \leq b_{i}\right\}$ for some $a_{i} \in X$ and $b_{i} \in \mathbb{R}, i \in I$, the following statements are equivalent for each $x_{0} \in D \cap \bigcap_{i \in I} C_{i}$ :

(i) $\left\{D, C_{i}: i \in I\right\}$ has the strong CHIP at each $x_{0}$.

(ii) For each $x \in X, P_{D \cap \bigcap_{i \in I} C_{i}}(x)=x_{0}$ if and only if there exists a finite set $I_{0} \subseteq I$ such that $P_{D}\left(x-\sum_{i \in I_{0}} a_{i}\right)=x_{0}$ 
where $P_{A}(x)$ denotes the projection of the point $x$ onto a convex set $A$. This important result has been extended in many aspects. For example, [32] discussed an extension to the case of an infinite system, and [31] discussed a family of functions in place of that of closed convex sets.

Recall that for a Banach space $X$ and its dual $X^{*}$, the duality map $\Phi: X \rightrightarrows X^{*}$ is defined by $\Phi(x):=\left\{x^{*}:\|x\|^{2}=\left\|x^{*}\right\|^{2}=\left\langle x^{*}, x\right\rangle\right\}$ (cf. [40, Section 3.7]). Let $\left\{g_{i}: i \in I\right\}, X, C, S$ and $I$ be as in section 2 .

Theorem 7.1. Suppose $X$ is a Banach space and let $x_{0} \in C \cap S$. Consider the following statements:

(i) $\left\{\delta_{C} ; g_{i}, i \in I\right\}$ satisfies the $B C Q$ at $x_{0}$.

(ii) For each $x \in X, x_{0} \in P_{C \cap S}(x)$ if and only if

$$
\Phi\left(x-x_{0}\right) \cap\left(N_{C}\left(x_{0}\right)+\text { cone } \bigcup_{i \in I\left(x_{0}\right)} \partial g_{i}\left(x_{0}\right)\right) \neq \emptyset .
$$

Then (i) $\Rightarrow$ (ii). If we further assume that $X$ is reflexive and smooth, then (ii) $\Leftrightarrow(\mathbf{i})$.

Proof. We regard the family $\left\{\delta_{C} ; g_{i}, i \in I\right\}$ as $\left\{g_{j}: j \in J\right\}$ by letting $J=I \cup\left\{i_{+}\right\}$and $g_{j}:=\delta_{C}$, where $i_{+} \notin I$. It follows that

$$
J\left(x_{0}\right):=\left\{j \in J: g_{j}\left(x_{0}\right)=\max \left\{\sup _{i \in I} g_{i}\left(x_{0}\right), \delta_{C}\left(x_{0}\right)\right\}\right\}=\left\{i_{+}\right\} \cup I\left(x_{0}\right) .
$$

Suppose (i) holds and let $x \in X$. Note that $x_{0} \in P_{C \cap S}(x)$ if and only if $x_{0}$ minimizes the function $\frac{1}{2}\|\cdot-x\|^{2}$ over the set $\left\{x: \delta_{C}(x)=0, g_{i}(x) \leq 0, \forall i \in I\right\}$. Since $\Phi\left(x-x_{0}\right)=-\partial\left(\frac{1}{2}\|\cdot-x\|^{2}\right)\left(x_{0}\right)$ (cf. [40, Page 230]) and since the family $\left\{g_{j}: j \in J\right\}$ satisfies the BCQ at $x_{0}$, it follows from (2.3) and Theorem 5.1(iii) that $x_{0} \in P_{C \cap S}(x)$ if and only if

$$
\Phi\left(x-x_{0}\right) \cap\left(N_{C}\left(x_{0}\right)+\text { cone } \bigcup_{i \in I\left(x_{0}\right)} \partial g_{i}\left(x_{0}\right)\right)=\Phi\left(x-x_{0}\right) \cap\left(\text { cone } \bigcup_{j \in J\left(x_{0}\right)} \partial g_{j}\left(x_{0}\right)\right) \neq \emptyset .
$$

Thus (ii) holds.

Now we assume in addition that $X$ is reflexive and smooth, and turn to prove (ii) $\Rightarrow(\mathbf{i})$. By (3.9), we need to show that

$$
N_{C \cap S}\left(x_{0}\right) \subseteq N_{C}\left(x_{0}\right)+\text { cone } \bigcup_{i \in I\left(x_{0}\right)} \partial g_{i}\left(x_{0}\right) .
$$

To do this, take $y^{*} \in N_{C \cap S}\left(x_{0}\right)$. By the given assumptions, $\Phi$ is bijective (cf. [40, Theorem 3.7.2 (vi) and Page 230]). Thus there exists $u=\Phi^{-1}\left(y^{*}\right)$ and it follows that $x_{0} \in P_{C \cap S}\left(x_{0}+u\right)$ by a well-known result (cf. [40, Corollary 3.8.5]). Therefore, by (ii), (7.1) holds with $x=x_{0}+u$. Thus we obtain from (7.1) that

$$
y^{*}=\Phi\left(x_{0}+u-x_{0}\right) \in N_{C}\left(x_{0}\right)+\text { cone } \bigcup_{i \in I\left(x_{0}\right)} \partial g_{i}\left(x_{0}\right) .
$$

This shows that (7.2) holds as required to show. This completes the proof.

Corollary 7.1. Suppose that $X$ in Theorem 7.1 is a Hilbert space and let $x_{0} \in C \cap S$. Then the following statements are equivalent:

(i) $\left\{\delta_{C} ; g_{i}, i \in I\right\}$ satisfies the $B C Q$ at $x_{0}$.

(ii) For each $x \in X, x_{0}=P_{C \cap S}(x)$ if and only if

$$
x-x_{0} \in N_{C}\left(x_{0}\right)+\text { cone } \bigcup_{i \in I\left(x_{0}\right)} \partial g_{i}\left(x_{0}\right) .
$$


(iii) For each $x \in X, x_{0}=P_{C \cap S}(x)$ if and only if there exist finite subset $J \subseteq I\left(x_{0}\right), \lambda_{i} \geq 0, u_{i} \in$ $\partial g_{i}\left(x_{0}\right), i \in J$ such that

$$
x_{0}=P_{C}\left(x-\sum_{i \in J} \lambda_{i} u_{i}\right)
$$

Proof. The equivalence of (ii) and (iii) is standard in Hilbert spaces. The equivalence of (i) and (ii) follows from Theorem 7.1 since (7.1) and (7.3) are now identical (because $\Phi$ is the identity map for Hilbert spaces)

Corollary 7.2. Suppose in Corollary 7.1 that the family $\left\{\delta_{C} ; g_{i}, i \in I\right\}$ has the conical EHP. Then for each $x \in X$ and $x_{0} \in C \cap S, P_{C \cap S}(x)=x_{0}$ if and only if there exist a finite set $I_{0} \subseteq I\left(x_{0}\right), x_{i} \in \partial g_{i}(x)$ and $\lambda_{i} \geq 0$ for each $i \in I_{0}$ such that $P_{C}\left(x-\sum_{i \in I_{0}} \lambda_{i} x_{i}\right)=x_{0}$.

Proof. Since the family $\left\{\delta_{C} ; g_{i}, i \in I\right\}$ has the conical EHP, it satisfies the BCQ by Theorem 4.1(ii). The result now follows from Corollary 7.1.

For the next two corollaries, let $g, C$, and $K$ be as in Example 2.1. These two corollaries were established respectively in [28] and [29] but under the additional assumption that $g$ is continuous.

Corollary 7.3. Suppose $X$ is a Hilbert space and let $x_{0} \in C \cap g^{-1}(-K)$. Then the following statements are equivalent:

(i) $\left\{\delta_{C}, \lambda g: \lambda \in K^{\oplus}\right\}$ satisfies the $B C Q$ at $x_{0}$.

(ii) The pair $\left\{C, g^{-1}(-K)\right\}$ has the sharpened strong CHIP at $x_{0}$, that is

$$
N_{C \cap g^{-1}(-K)}\left(x_{0}\right)=N_{C}\left(x_{0}\right)+\bigcup_{\substack{\lambda \in K^{\oplus} \\ \lambda g\left(x_{0}\right)=0}} \partial(\lambda g)\left(x_{0}\right) .
$$

(iii) For each $x \in X, x_{0}=P_{C \cap g^{-1}(-K)}(x)$ if and only if $x_{0}=P_{C}(x-l)$ for some $l \in \bigcup_{\substack{\lambda \in K^{\oplus} \\ \lambda g\left(x_{0}\right)=0}} \partial(\lambda g)\left(x_{0}\right)$.

Proof. By Fact 6.3 and Remark 6.1, (i) $\Leftrightarrow($ ii). The equivalence (i) $\Leftrightarrow$ (iii) follows from Corollary 7.1 as applied to $I:=K^{\oplus}$ and $g_{\lambda}:=\lambda g$ (so $I\left(x_{0}\right)=\left\{\lambda \in K^{\oplus}: \lambda g\left(x_{0}\right)=0\right\}$ ).

Corollary 7.4. Suppose $X$ is a Hilbert space and that (C') holds. Let $x_{0} \in C \cap g^{-1}(-K)$ and $x \in X$. Assume that $\left\{C, g^{-1}(-K)\right\}$ has the strong CHIP at $x_{0}$. Then the following statements are equivalent:

(i) $x_{0}=P_{C \cap g^{-1}(-K)}(x)$.

(ii) $x_{0}=P_{C}(x-l)$ for some $l \in \bigcup_{\substack{\lambda \in K^{\oplus} \\ \lambda g\left(x_{0}\right)=0}} \partial(\lambda g)\left(x_{0}\right)$.

Proof. By Corollary 6.3, the given assumptions imply that $\left\{C, g^{-1}(-K)\right\}$ has the sharpened strong CHIP at $x_{0}$. By the implication (ii) $\Rightarrow$ (iii) in the preceding corollary, the result is now clear.

Acknowledgement. The authors would like to express their sincere thanks to the three anonymous referees for many helpful comments and for pointing out the references [1, 4-6, 12, 16, 17, 37].

\section{References}

[1] M. Ait Mansoour, A. Metrane and M. Théra, Lower semicontinuous regularization for vector-valued mappings, J. Global Optim., 35 (2006), no. 2, pp. 283-309.

[2] V. Barbu and T. Precupanu, Convexity and Optimization in Banach Spaces, Sijthoff \& Noordhoff, the Netherlands, 1978. 
[3] J. Borwein and R. Goebel, Notions of relative interior in Banach spaces, Optimization and related topics, J. Math. Sci., 115 (2003), no. 4, pp.2542-2553.

[4] R. I. Bot,, S. M. Grad and G. Wanka, A new constraint qualification for the formula of the subdifferential of composed convex functions in infinite dimensional spaces, to appear in Math. Nachr..

[5] R. I. Bot, S. M. Grad and G. Wanka, On strong and total Lagrange duality for convex optimization problems, to appear in J. Math. Anal. Appl..

[6] R. I. Bot, S. M. Grad and G. Wanka, A weaker regularity condition for subdifferential calculus and Fenchel duality in infinite dimensional spaces, Nonlinear Anal., 64(2006), pp. 2787-2804.

[7] R. I. Boţ and G. Wanka, An alternative formulation for a new closed cone constraint qualification, Nonlinear Anal., 64 (2006), pp. 1367-1381.

[8] R. S. Burachik and V. Jeyakumar, A simple closure condition for the normal cone intersection formula, Proc. Amer. Math. Soc., 133(2005), pp. 1741-1748.

[9] R. S. Burachik and V. Jeyakumar, A new geometric condition for Fenchel's duality in infinite dimensional spaces, Math. Program., Ser. B, 104(2005), pp. 229-233.

[10] R. S. Burachik and V. Jeyakumar, A dual condition for the convex subdifferential sum formula with applications, J. Convex Anal., 12(2005), pp. 279-290.

[11] F. Clarke, Optimization and Nonsmooth Analysis, John Wiley \& Sons, Inc., New York, 1983.

[12] C. Combari, M. Laghdir and L. Thibault, Sous-différentiels de foncitions convexes composées, Ann. Sci. Math. Quebec, 18 (1994), pp. 119-148.

[13] F. Deutsch, W. Li and J. Ward, A dual approach to constrained interpolation from a convex subset of Hilbert space, J. Approx. Theory, 90(1997), pp. 385-414.

[14] F. Deutsch, W. Li and J. Ward, Best approximation from the intersection of a closed convex set and a polyhedron in Hilbert space, weak Slater conditions, and the strong conical hull intersection property, SIAM J. Optim., 10(1999), pp. 252-268.

[15] N. Dinh, M. A. Goberna and M. A. López, From linear to convex systems: consistency, Farkas' lemma and applications, J. Convex Anal., 13(2006), pp. 113-133.

[16] N. Dinh, M. A. Goberna, M. A. López and T. Q. Son, New Farkas-type constaint qualification in convex semi-infinite programming, ESAIM, Control, Optimization and Calculus of Variations, 13(2007), pp. 580-597.

[17] M. A. Goberna, V. Jeyakumar and M. A. López, Necessary and sufficient conditions for solvability of systems of infinite convex inequalities, Nonlinear Anal., doi: 10.1016 / j.na. 2006.12.014.

[18] M. A. Goberna and M. A. López, Linear Semi-Infinite Optimization, Wiley Series in Mathematical Methods in Practice, Wiley, Chichester, 1998.

[19] J. Hiriart-Urruty and C. Lemarechal, Convex Analysis and Minimization Algorithms I, Vol. 305 of Grundlehren der Mathematschen Wissenschaften, Springer-verlag, New York, 1993.

[20] R. B. Holmes, Geometric Functional Analysis and its Applications, Springer-Verlag, New York, 1975.

[21] H. Hu, Characterizations of local and global error bounds for convex inequalities in Banach spaces, SIAM J. Optim., 18(2007), pp. 309-321. 
[22] J. Jahn, Mathematical Vector Optimization in Partially Ordered Linear Spaces, Methoden und verfahren der mathematischen Physik, Band 31, Verlag Peter Lang, 1986.

[23] V. Jeyakumar, Characterizing set containments involving infinite convex constraints and reverseconvex constraints, SIAM J. Optim., 13(2003), pp. 947-959.

[24] V. Jeyakumar, The strong conical hull intersection property for convex programming, Math. Program., Ser. A, 106(2006), pp. 81-92.

[25] V. Jeyakumar, N. Dinh and G. M. Lee, New sequential Lagrange multiplier conditions characterizing optimality without constraint qualification for convex programs, SIAM J. Optim., 14(2003), pp. 534547.

[26] V. Jeyakumar, N. Dinh and G. M. Lee, A new closed cone constraint qualification for convex optimization, Applied Mathematics Research Report AMR 04/6, University of New South Wales, 2004.

[27] V. Jeyakumar, N. Dinh and G. M. Lee, Liberating the subgradient optimality conditions from constraint qualifications, Applied Mathematics Research Report AMR 04/7, University of New South Wales, 2004.

[28] V. Jeyakumar and H. Mohebi, Limiting $\epsilon$-subgradient characterizations of constrained best approximation, J. Approx. Theory, 135 (2005), pp. 145-159.

[29] V. Jeyakumar and H. Mohebi, A global approach to nonlinearly constrained best approximation, Numer. Funct. Anal. Optim., 26 (2005), pp. 205-227.

[30] V. Jeyakumar, A. M. Rubinov, B. M. Glover and Y. Ishizuka, Inequality systems and global optimization, J. Math. Anal. Appl., 202 (1996), no. 3, pp. 900-919.

[31] C. Li and X. Q. Jin, Nonlinearly constrained best approximation in Hilbert spaces, the strong CHIP and the basic constraint qualification condition, SIAM J. Optim., 13(2002), pp. 228-239.

[32] C. Li and K. F. Ng, Constraint qualification, the strong CHIP and best approximation with convex constraints in Banach spaces, SIAM J. Optim., 14(2003), pp. 584-607.

[33] C. Li and K. F. Ng, On constraint qualification for infinite system of convex inequalities in a Banach space, SIAM J. Optim., 15(2005), pp. 488-512.

[34] C. Li and K. F. Ng, Strong CHIP for infinite system of closed convex sets in normed linear spaces, SIAM J. Optim., 16(2005), pp. 311-340.

[35] C. Li, K. F. Ng and T. K. Pong, The SECQ, linear regularity and the strong CHIP for infinite system of closed convex sets in normed linear spaces, SIAM J. Optim., 18(2007), pp. 643-665.

[36] W. Li, C. Nahak and I. Singer, Constraint qualifications for semi-infinite systems of convex inequalities, SIAM J. Optim., 11(2000), pp. 31-52.

[37] J. P. Penot and M. Théra, Semi-continuous mappings in general topology, Arch. Math., 38(1982), pp.158-166.

[38] H. L Royden, Real Analysis, Third edition, Pearson Edu., Inc., 1988.

[39] I. Singer, Best Approximation in Normed Linear Spaces by Elements of Linear Spaces, Die Grundlehren der mathematischen Wissenschaften in Einzeldarstellungen, Band 171 ,SpringerVerlag, 1970.

[40] C. Zălinescu, Convex Analysis in General Vector Spaces, World Scientific (2002). 